\title{
Aberration and the Fundamental Speed of Gravity in the Jovian Deflection Experiment
}

\author{
Sergei M. Kopeikin* \\ Department of Physics \& Astronomy, University of Missouri-Columbia, Columbia, MO 65211, USA \\ Edward B. Fomalont \\ National Radio Astronomy Observatory, Charlottesville, VA 22903, USA
}

\begin{abstract}
We describe our explicit Lorentz-invariant solution of the Einstein and null geodesic equations for the deflection experiment of 2002 September 8 when a massive moving body, Jupiter, passed within 3.7 ' of a line-of-sight to a distant quasar. We develop a general relativistic framework which shows that our measurement of the retarded position of a moving light-ray deflecting body (Jupiter) by making use of the gravitational time delay of quasar's radio wave is equivalent to comparison of the relativistic laws of the Lorentz transformation for gravity and light. Because, according to Einstein, the Lorentz transformation of gravity field variables must depend on a fundamental speed $c$, its measurement through the retarded position of Jupiter in the gravitational time delay allows us to study the causal nature of gravity and to set an upper limit on the speed of propagation of gravity in the near zone of the solar system as contrasted to the speed of the radio waves. In particular, the $v / c$ term beyond of the standard Einstein's deflection, which we measured to $20 \%$ accuracy, is associated with the aberration of the null direction of the the gravity force ("aberration of gravity") caused by the Lorentz transformation of the Christoffel symbols from the static frame of Jupiter to the moving frame of observer. General relativistic formulation of the experiment identifies the aberration of gravity with the retardation of gravity because the speed of gravitational waves in Einstein's theory is equal to the speed of propagation of the gravity force. We discuss the misconceptions which have inhibited the acceptance of this interpretation of the experiment. We also comment on other interpretations of this experiment by Asada, Will, Samuel, Pascual-Sánchez, and Carlip and show that their "speed of light" interpretations confuse the Lorentz transformation for gravity with that for light, and the fundamental speed of gravity with the physical speed of light from the quasar. For this reason, the "speed of light" interpretations are not entirely consistent with a retarded LiénardWiechert solution of the Einstein equations, and do not properly incorporate how the phase of the radio waves from the quasar is perturbed by the retarded gravitational field of Jupiter. Although all of the formulations predict the same deflection to the order of $v / c$, our formulation shows that the underlying cause of this deflection term is associated with the aberration of gravity and not of light, and that the interpretations predict different deflections at higher orders of $v / c$ beyond the Shapiro delay, thus, making their measurement highly desirable for deeper testing of general relativity in future astrometric experiments like Gaia, SIM, and SKA.
\end{abstract}

PACS numbers: 04.20.-q, 04.80.Cc

Keywords: general relativity - experimental gravity - speed of gravity - speed of light

*Electronic address: kopeikins@missouri.edu

${ }^{\dagger}$ Electronic address: efomalon@nrao.edu 
Contents

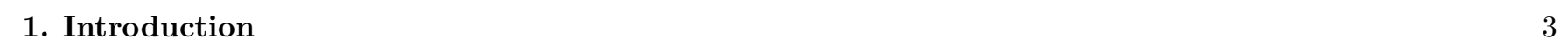

2. The Metric Tensor, Retarded Time and the Fundamental Speed 4 2.1. Formulation 4

2.2. Interpretation of the Einstein equations 6

2.3. The gravito-magnetic field 6

$\begin{array}{ll}2.4 \text {. The speed of gravitv and the Newtonian limit } & 7\end{array}$

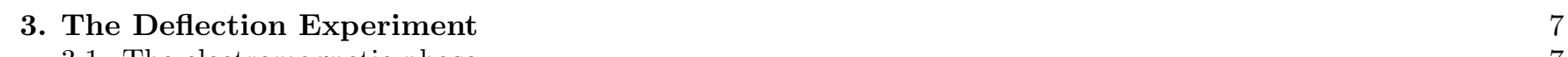

3.1. The electromagnetic phase $\quad 7$

$\begin{array}{ll}\text { 3.2. The gravitational time delav } & 8\end{array}$

$\begin{array}{ll}\text { 3.3. The aberration of gravity } & 9\end{array}$

3.4. Why can VLBI determine the retarded position of Jupiter? 10

3.5. Fundamental speed of gravitv as a fitting parameter 11

3.6. The determination of the speed of gravity 12

4. Common Misconceptions Associated with This Experiment 13

4.1. Misconception 1: The gravitational field of a uniformlv moving bodv is statid 13

4.2. Misconception 2: The propagation of the gravitational field of a uniformlv moving bodv can not be observed 14

4.3. Misconception 3: The parameter $c$ of the Lorentz transformation is phvsically the speed of light 14

4.4. Misconception 4: The speed of gravitv can onlv be measured through observation of gravitational waves 14

4.5. Misconception 5: The retarded position of Jupiter is taken at the time of the light-rav closest approach 15

4.6. Misconception 6: Null characteristics of gravitv field can be observed onlv at $(v / c)^{2}$ terms bevond the Shapiro delav 15

4.7. Misconception 7: The Jovian experiment and the PPN parameter $\alpha_{1} .17$

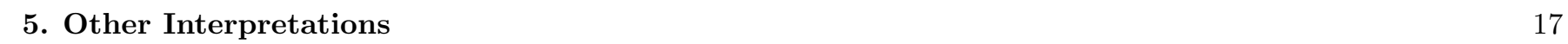

\begin{tabular}{|lr}
\hline 6. Summary & 18
\end{tabular}

\begin{tabular}{|lr}
\hline A. Propagation of Light in a Bi-metric Theorv & 19
\end{tabular}

$\begin{array}{ll}\text { References } & 22\end{array}$ 


\section{INTRODUCTION}

The recent Very Long Baseline Interferometric (VLBI) experiment of the ultra-precise measurement of the relativistic deflection of a quasar's radio waves (light) by the gravitational field of Jupiter [1, 2] has captivated the keen interest of relativists who were trying to deeply understand gravitational physics. The experiment and its physical interpretation has illustrated some of the important subtleties in the understanding, the approaches, and the mathematical techniques of general relativity (GR).

The experimental goal was the measurement of the (non-radial or tangential) deflection component associated with the retarded position of Jupiter caused by its orbital motion and mathematically connected to the finite value of the fundamental speed entering in front of the time derivatives from the metric tensor in the gravity field and null geodesic equations (that is, the fundamental constant $c$ in the Christoffel symbols and the curvature tensor). This fundamental speed of gravity causes the retardation in propagation of gravity force from a moving massive object which can be measured in the light-ray deflection experiments. In this paper we use general theory of relativity and denote the fundamental speed of gravity by $c$. Label $c_{g}$ for the speed of gravity is understood as a parameter in the experimental fitting of observations to the Einstein theory. The symbol $c$ is frequently called as the speed of light. However, one must clearly separate the relativistic effects caused by the physical speed of light from those associated with the fundamental speed of gravity in the Eiunstein equations. We shall show in the present paper how to distinguish these effects to avoid ambiguity.

Einstein's general principle of relativity assumes that the gravity field obeys to the special principle of relativity and transforms from one inertial frame to another in accordance with the Lorentz transformation of the Minkowski space-time. Hence, it postulates that in a geometrized system of units [3] the speed of gravity $c=1$ in any frame of reference. We measured the impact of the retarded gravitational field of Jupiter on light deflection (as well as the instantaneous Shapiro deflection of $1100 \mu \mathrm{arcsec}$ ) of $50 \pm 9 \mu \mathrm{arcsec}$, in excellent agreement with the GR prediction of $51 \mu$ arcsec based on assumption that $c=1$ irrespectively of the reference frame. We have interpreted these result as a measurement of the fundamental speed of gravity $c=1.06 \pm 0.21$ and confirmation that gravity interacts with moving particles (photons) not instantaneously.

However, at present there are four other interpretations, which do not agree among themselves, concerning the results of the experiment ${ }^{1}$.

- the experiment measured the speed of the radio waves from the quasar in the static gravitational field of Jupiter [5],

- the experiment measured the aberration of the radio waves from the quasar [6, 7],

- the experiment measured no significant property of light or of gravity 8],

- the experiment measured the light-time delay discovered by Rømer in $1676[9]$

In this paper, we streamline our previous theoretical calculations by working out an explicit Lorentz-invariant Liénard-Wiechert solution of the Einstein gravity field equations in $\S 2$. The VLBI measurement of the phase of a radio wave, which has propagated in the field of a moving body (Jupiter), is described in $\S 3$. We demonstrate that the fundamental speed of the characteristics of the gravity field equations (which we call for brevity "the speed of gravity") and the aberration of gravity force are indeed associated with the experiment. The major misconceptions about the experiment and properties of gravity in general relativity are summarized in $\S 4$. Finally, in $\S 5$ we outline why these alternative interpretations of the experimental results differ with our general relativistic interpretation, even though all interpretations predict the same angular deflection result to order $v / c$ beyond the Shapiro delay. Appendix describes how light propagates in a simple bi-metric model of gravity with two metrics, and provides a clear evidence that a light-ray deflecting body deflects light from its retarded position connected to observer by a null line lying on the gravity null cone that is because gravity propagates with finite speed.

\footnotetext{
${ }^{1}$ Recent publication of Frittelli [4] agrees with our understanding of the gravitational physics of the Jovian deflection experiment
} 


\section{THE METRIC TENSOR, RETARDED TIME AND THE FUNDAMENTAL SPEED}

\subsection{Formulation}

Our interpretation of the experiment is based solely on General Relativity and the physical meaning of the retarded Liénard-Wiechert potentials used to solve the Einstein equations. These potentials describe the propagation of the gravity field even in the case of gravitating bodies which move uniformly with constant speed 10 exactly as in electrodynamics where the retarded Liénard-Wiechert potentials describe electromagnetic field of a moving point charge 11, 12. We emphasize that although gravitational waves, which amplitude is falling off as $1 / r$, are not generated by a uniformly moving body the null characteristics of the gravitational field are precisely defined by the Liénard-Wiechert potentials through their property of the Lorentz invariance and the principle of physical causality. Thus, our use of the "speed of gravity" term is a synonym for the ultimate speed of the null characteristics of the gravity field equations. This speed is numerically equal to the fundamental speed of the Minkowski geometry which is associated with the speed of light in vacuum. However, in general relativity the fundamental speed could be different from the speed of light. In the near-field zone of a gravitating system the null characteristics of gravity reveal themselves through the Lorentz invariance of the gravitational force (the aberration of gravity) when one compares observations done in one inertial frame with another, while in the far-field radiative zone the null characteristics of gravity can be traced as freely propagating gravitational waves.

In general relativity light and gravity null rays are bi-characteristics, that is they propagate with the same fundamental speed. At the first glance it may look that they are identical and can not be discriminated. However, this point of view is erroneous. Although light and gravity propagate with the same speed on the hyper-surface of one and the same null cone, they (1) generally propagate in different directions in space in each particular gravitational experiment, and (2) they are associated with different physical effects (the aberration of light and the aberration of gravity, for instance). Hence, discrimination between the light and gravity null cones is possible if one makes use of additional properties of the propagating system of the light and gravity null rays.

Our definition of the "speed of gravity" is more general than that used by Asada, Samuel, and Will 5, 6, 8] who limited its meaning as the speed of propagation of free gravitational waves alone. In their formulations of the experiment, these authors assumed only far-field gravitational effects, where gravitational waves are dominant and, hence, the differentiation between the speed of gravity $c$ and that of light occurs only at orders of $(v / c)^{2}$ beyond the Shapiro delay and higher. This was one reason why the "speed of light" was interpreted as causing the observed aberration of gravity force of Jupiter. However, the experiment was performed in the near-field of the quasar radio wave-Jupiter interaction where the transformational degrees of freedom for gravity not associated with gravitational waves are dominant but they must not be confused with the transformational degrees of freedom for radio waves used for observations.

It is clear that there are different theoretical frameworks to interpret the Jovian deflection experiment as a new test of non-stationary properties of the gravitational field in the solar system. However, our point of view is that the most natural interpretation should rest solidly on GR which has passed all other experimental tests in strong compliance with our current theoretical understanding of gravitational physics. In other words, in order to interpret the experiment properly, the space-time properties which GR postulates must not be violated; that space-time is a differentiable manifold endowed with an affine connection (Christoffel symbols) whose geodesics form a privileged set of world-lines in space-time, and their knowledge (observation) allows us to extract information about the curvature tensor (tidal gravitational force) without ambiguity. The Christoffel symbols define the law of motion of test particles and extended bodies and, hence, generalize the concept of the Newtonian gravitational force in GR. They are formed from partial derivatives of the metric tensor that defines geometric properties of the space-time and the causal structure of the null cone. At the same time the metric tensor is associated with a gravitational potential whose properties are determined by Einstein's equations.

In what follows the Greek indices $\alpha, \beta, \ldots$ run from 0 to 3 , the Roman indices $i, j, \ldots$ run from 1 to 3 , repeated Greek indices mean Einstein's summation from 0 to 3 , and bold letters $\boldsymbol{a}=\left(a^{1}, a^{2}, a^{3}\right)$, $\boldsymbol{b}=\left(b^{1}, b^{2}, b^{3}\right)$, etc. denote spatial (3-dimensional) vectors. A dot between two spatial vectors, for example $\boldsymbol{a} \cdot \boldsymbol{b}=a^{1} b^{1}+a^{2} b^{2}+a^{3} b^{3}$, means the Euclidean dot product, and the cross between two vectors, for example $\boldsymbol{a} \times \boldsymbol{b}$, means the Euclidean cross product. We also use a shorthand notation for partial derivatives $\partial_{\alpha}=\partial / \partial x^{\alpha}$. Greek indices are raised and lowered with full metric $g_{\alpha \beta}$. The Minkowski (flat) space-time metric $\eta_{\alpha \beta}=\operatorname{diag}(-1,+1,+1,+1)$.

The solution of the Einstein equations, using the Liénard-Wiechert potentials has been essentially understood and developed by previous researchers and we do not intend to go into complete details of this iterative procedure called by the post-Minkowskian approximations [13]. For our purposes the first (linearized gravity) post-Minkowskian approximation is sufficient since the gravitational light-ray deflection experiments are not yet sensitive enough to measure non-linearities of the Einstein equations. The linearized gravity approximation is as follows. 
We introduce the weak-field decomposition of the metric tensor

$$
g_{\alpha \beta}=\eta_{\alpha \beta}+h_{\alpha \beta},
$$

where $h_{\alpha \beta}$ is the post-Minkowskian perturbation of the Minkowski metric tensor $\eta_{\alpha \beta}$. We impose the harmonic gauge condition [3] on the metric tensor

$$
\partial_{\alpha} h^{\alpha \beta}-\frac{1}{2} \partial^{\beta} h_{\lambda}^{\lambda}=0
$$

The linearized Einstein equations in the first post-Minkowski approximation are of the hyperbolic-type (wave) equations for the metric perturbation. In arbitrary harmonic coordinates $x^{\alpha}=(c t, \boldsymbol{x})$, the Einstein equations read ${ }^{2}$

$$
\square h^{\mu \nu}=-\frac{16 \pi G}{c^{4}}\left(T^{\mu \nu}-\frac{1}{2} \eta^{\mu \nu} T_{\lambda}^{\lambda}\right) .
$$

Here $\left(\square=-c^{-2} \partial^{2} / \partial t^{2}+\nabla^{2}\right)$ is the wave operator in flat space-time, and $T^{\mu \nu}$ is the stress-energy tensor of point-like light-ray deflecting bodies in linearized approximation

$$
T^{\mu \nu}(t, \boldsymbol{x})=\sum_{a=1}^{N} M_{a} \gamma_{a}^{-1}(t) u_{a}^{\mu}(t) u_{a}^{\nu}(t) \delta^{(3)}\left(\boldsymbol{x}-\boldsymbol{x}_{a}(t)\right),
$$

where the index $a=1,2, \ldots, N$ enumerates gravitating bodies of the solar system, $M_{a}$ is the (constant) rest mass of the ath body, $\boldsymbol{x}_{a}(t)$ is time-dependent spatial coordinate of the $a$ th body, $\boldsymbol{v}_{a}(t)=d \boldsymbol{x}_{a}(t) / d t$ is velocity of the $a$ th body, $u_{a}^{\alpha}=\gamma_{a}\left(1, \boldsymbol{v}_{a} / c\right)$ is the four-velocity of the $a$ th body, $\gamma_{a}=\left(1-v_{a}^{2} / c^{2}\right)^{-1 / 2}$ is the Lorentz-factor, and $\delta^{(3)}(\boldsymbol{x})$ is the 3 -dimensional Dirac's delta-function. We have neglected $\sqrt{-g}$ in Eq. (2.4) because in the linearized approximation $\sqrt{-g}=1+O(h)$ and the terms proportional to the metric tensor perturbation are irrelevant in $T^{\mu \nu}$.

Because the Einstein equations (2.3) are linear, we can consider their solution as a linear superposition of the solutions for each body. It allows us to focus on the relativistic effects caused by one body (Jupiter) only, although in the actual experiment the gravitational fields of Sun and Earth are also important and were included in the analysis (see 2, 14] for more detail). Solving Einstein's equations (2.3) by making use of the retarded Liénard-Wiechert tensor potentials [15], one obtains the post-Minkowski metric tensor perturbation for Jupiter [15, 16]

$$
h^{\mu \nu}(t, \boldsymbol{x})=\frac{2 G M_{J}}{c^{2}} \frac{2 u^{\mu} u^{\nu}+\eta^{\mu \nu}}{r_{R}}
$$

where $r_{R} \equiv-u_{\alpha} r^{\alpha}, r^{\alpha}=x^{\alpha}-x_{J}^{\alpha}(s)$. The metric perturbations for Sun and Earth look similar to Eq. (2.5) with a corresponding replacement of masses, coordinates and velocities for the Sun and Earth respectively. In Eq. (2.5), $M_{J}$ is the mass of Jupiter, $u^{\alpha}=c^{-1} d x_{J}^{\alpha}(s) / d \tau=\gamma(s)\left(1, \boldsymbol{v}_{J}(s) / c\right)$ is its four-velocity, with $\tau=\tau(s)$ being a proper time on Jupiter's world line, $\boldsymbol{v}_{J}(s)=d \boldsymbol{x}(s) / d s$ is Jupiter's coordinate velocity, and $\gamma(s)=d s / d \tau=\left(1-v_{J}^{2}(s) / c^{2}\right)^{-1 / 2}$. Notice that the metric tensor perturbation (2.5) is valid for accelerated motion of Jupiter and is not restricted by the approximation of a body moving on a straight line (see [15] for more detail). In other words, the four-velocity $u^{\alpha}$ in Eq. (2.5) is not a constant.

Because we solved the Einstein equations (2.3) in terms of the retarded Liénard-Wiechert potentials, the distance $r^{\alpha}=x^{\alpha}-x_{J}^{\alpha}(s)$, the Jupiter's worldline $x_{J}^{\alpha}(s)=\left(c s, \boldsymbol{x}_{J}(s)\right)$, and the four-velocity $u^{\alpha}(s)$ are all functions of the retarded time $s$ [15]. The retarded time $s$ is found in the first post-Minkowski approximation as a solution of the gravity null cone equation

$$
\eta_{\mu \nu} r^{\mu} r^{\nu} \equiv \eta_{\mu \nu}\left(x^{\mu}-x_{J}^{\mu}(s)\right)\left(x^{\nu}-x_{J}^{\nu}(s)\right)=0
$$

that is

$$
s=t-\frac{1}{c}\left|\boldsymbol{x}-\boldsymbol{x}_{J}(s)\right|,
$$

where the fundamental constant $c$ in Eq. (2.7) must be conceptually understood as the speed of characteristics of the gravity field equations because it comes from the wave operator of the Einstein gravity field Eqs. (2.3). Indeed,

\footnotetext{
${ }^{2} t$ is time and $\boldsymbol{x}$ is space coordinates.
} 
Maxwell equations have not yet been considered; hence the retardation in Eq. (2.7) cannot be associated with the propagation of electromagnetic waves (light). In what follows, the retarded time $s$ will appear in all subsequent equations related to the propagation of radio waves. It is important to keep in mind that Eq. (2.7) connects the point of observation $\boldsymbol{x}$ and the retarded position of Jupiter $\boldsymbol{x}(s)$ by a null line which is always a characteristic of the Einstein field equations. Radio waves are also propagating along a null direction connecting the observer and the source of radio waves (quasar). However, the null characteristic of the Einstein equations (2.7) is well separated in the sky from the null direction associated with the propagation of the radio wave so that they can not be observationally confused (see Appendix A for additional details).

\subsection{Interpretation of the Einstein equations}

The retardation shown in Eqs. 2.5 - 2.7) is physically associated with the finite speed of propagation of the null characteristics of the gravity field. In the most general case, this propagation is realized in the form of a traveling gravitational field which can be decomposed into components of different algebraic types [17]. In the wave zone of the solar system, the traveling gravitational field decouples from the system and propagates as a transverse-traceless (TT) gravitational wave with amplitude decaying as $1 / r$ where $r$ is the distance from the system. However, in the near and transition zones of the solar system, a propagating gravitational field has components which are not completely decoupled from the matter and decay faster than $1 / r[17]$. These components of the propagating gravitational field are closely associated with the residual gauge freedom of the metric tensor $g_{\alpha \beta}$ and for this reason we call them the gauge modes. They are also known as transverse (T) and longitudinal (L) wave modes [3]. These gauge modes control the validity of the laws of conservation of matter and gravity field in the lower order post-Newtonian approximations [10, 18].

All components of the propagating gravitational field of the solar system interact with radio waves moving from the quasar to the Earth, and perturb the phase of an electromagnetic waves according to the retardation Eq. (2.7) which incorporates both the near-field and far-field effects of gravity as explained in $\S 3$. The use of the retarded Liénard-Wiechert potentials, rather than the advanced potentials, is consistent with the principle of causality, and the observation of the orbital decay of binary pulsars caused by emission of gravitational radiation [19].

The speed $c$ is a fundamental speed of the special theory of relativity and Lorentz-invariance of flat (Minkowski) space-time metric $\eta_{\alpha \beta}$. According to our definition the speed of gravity is a fundamental constant of the curved spacetime manifold which is associated with temporal variation of gravitational field and appears as a physical quantity any time when one takes a derivative of the metric tensor with respect to time coordinate $x^{0}$ [21]. In GR the speed of gravity is equal to $c$ because at each point the curved space-time manifold has a tangent space-time which coincides with Minkowski space. But this postulate must be tested experimentally [20, 21].

The measured Jovian deflection is most sensitive to the retardation of the gravitational field caused by its Lorentz invariant properties. Thus, what we measured was not the speed of TT gravitational waves but the speed of gravity in the near-field zone. Since the speed of TT gravitational waves can not exceed the speed of gravity in the near-zone for any viable theory of gravity [22], our VLBI experiment sets an upper limit on the speed of propagation of gravitational waves as well.

\subsection{The gravito-magnetic field}

Einstein's equations in the frame attached to Jupiter do not contain time derivatives of the metric tensor because the Jovian field is static; hence the retardation of gravity (associated with time derivatives of the metric tensor and its null characteristics) cannot be measured in this frame. This condition has led many to infer that the null characteristics and retardation of the gravity field must remain unmeasurable after a Lorentz transformation to another frame, for example to the surface of the earth or the barycenter of the solar system. Thus, it is claimed, that the deflectionof-light measurement cannot be associated with the retardation of gravity. But, the Lorentz transformation of the Einstein equations (2.3) from static to a moving frame induces time derivatives of the metric tensor perturbations which can be measured in the light deflection experiments from the retardation of gravity field [2] in order to determine the fundamental speed of its null characteristics.

Our interpretation does not mean that the laws of gravitational physics change under a Lorentz transformation, but only that some property of gravity can become measurable after transformation from a static to a non-static frame. A similar example comes from electrodynamics in the case of a single electron which has a static electric field in its own frame. A Lorentz transformation to a moving frame induces a new field - the magnetic field, which was not observable in the static frame. Maxwell's equations, also, show that the strength of the magnetic field in the non-static frame depends on a time derivative of the electric field coupled with the speed of light. 
The same analogy is associated with the Jovian deflection experiment. In Jupiter's frame there is only a static "electric-type" gravitational field. With a Lorentz transformation to a moving frame, a "magnetic-type" field, called a gravito-magnetic field, is induced. This field, according to the theory of general relativity, arises from moving matter (matter currents) just as an ordinary magnetic field arises from moving charges (electrical currents). The analogy is so apt that the equations describing this "magnetic-type" component of gravity can essentially be adapted from Maxwell's equations for electromagnetism by replacing the charge density with the mass density and the charge current with the mass current, with changes of numerical factors reflecting different helicities of electromagnetic (spin 1) and gravitational (spin 2) fields 23]. Thus, in a moving frame, the translational motion of Jupiter produces the gravito-magnetic field which deflects the light tangentially by dragging it to the direction of motion of Jupiter [21, 24]. We can measure this tangential gravito-magnetic dragging of light and express its magnitude in terms of the speed of characteristics of the gravity field equations, in the analogous way that we can express the magnitude of the magnetic field interaction in terms of the speed of the characteristics of electromagnetic field equations [21, 24].

However, the predicted light deflection must be invariant to any Lorentz transformation. This is clearly the case because all of the calculations of the deflection experiment, regardless of the reference frame, predict the same deflection! But, the gravito-magnetic property of gravity that is sampled by the experiment does depend on the reference frame. In Jupiter's frame, all of the deflection is associated with the static gravitational field - but we do not observe in this frame. In the frame of the experiment (the barycentric of the solar system), the deflection is associated with a (radial) static gravitational field of Jupiter plus a small (tangential) gravito-magnetic field, due to its orbital velocity, which is coupled with the speed of gravity, and reveals itself through the retardation of gravity already in terms of order $v / c$ beyond the Shapiro delay. More precise measurements of the deflection by Jupiter, which are directly sensitive to TT gravitational waves, will require a more exact solution of the Einstein equations taking into account Jupiter's orbital acceleration.

\subsection{The speed of gravity and the Newtonian limit}

The Principle of Correspondence of GR with the Newtonian theory (closely related to the Principle of Equivalence) does not provide any information on the speed of gravity because the speed of gravity is associated with the temporal changes of the gravitational field while the Newtonian limit is stationary. In principle, the speed of gravity should appear in the right side of the Einstein equations as a constant characterizing coupling between space-time and matter variables [21]. Thus, it seems natural to state that the speed of gravity can be already derived from the validity of the Principle of Correspondence between Einstein's theory of general relativity and the Newtonian theory of gravity. But, the metric tensor perturbation in the Newtonian limit of general relativity depends on a coupling constant $\kappa=G / c^{2}$, where $G$ is the observed value of the universal gravitational constant. If the speed of gravity were different from $c$ the difference would be absorbed by re-definition of the observed value of $G$ in such a way that the coupling constant $\kappa$ is kept fixed [20, 21].

\section{THE DEFLECTION EXPERIMENT}

\subsection{The electromagnetic phase}

Let us assume that the speed of light is equal to the speed of gravity. It simplifies calculations but the observable effects of the light and gravity propagation can be still clearly separated as we discuss later. For the doubting reader appendix of this paper gives more general covariant description of propagation of light rays in a bi-metric theory of gravity, thus, helping one to formally discern null characteristics of light from those for gravity given by Eq. (2.7).

Very Long Baseline Interferometry (VLBI) measures the phase $\varphi$ of the wave front coming from a radio source. The phase is an invariant scalar function with respect to coordinate transformations, and it is determined in the approximation of geometric optics from the eikonal equation [3, 25]

$$
g^{\mu \nu} \partial_{\mu} \varphi \partial_{\nu} \varphi=0
$$

where $g^{\mu \nu}=\eta^{\mu \nu}-h^{\mu \nu}$. The eikonal Eq. (3.8) is a direct consequence of Maxwell's equations [3, 26, 31] and its solution describes a wave front of an electromagnetic wave propagating in curved space-time defined by the metric tensor in Eqs. (2.1), (2.5). We emphasize that the electromagnetic wave in Eq. (3.8) is a test field that has no back-action on the properties of the metric tensor perturbation $h_{\mu \nu}$, and does not change the curvature of the space-time. Hence, the structure of Eq. (3.8) is not related to the specific properties of the electromagnetic field, but is completely determined by the structure of space-time, or, more accurately, its geometry defined by Eqs. [2.1), (2.5) 26]. 
Let us introduce a co-vector $K_{\alpha}=\partial_{\alpha} \varphi$. Let $\lambda$ be an affine parameter along a light ray orthogonal to the electromagnetic wave front $\varphi$. The vector $K^{\alpha}=d x^{\alpha} / d \lambda=g^{\alpha \beta} \partial_{\beta} \varphi$ is tangent to the light ray. Eq. (3.8) expresses a simple fact that the vector $K^{\alpha}$ is null, that is $g_{\mu \nu} K^{\mu} K^{\nu}=0$. Thus, the light rays are null geodesics defined in the linearized post-Minkowskian approximation by equation [25]

$$
\frac{d K_{\alpha}}{d \lambda}=\frac{1}{2} \partial_{\alpha} h_{\mu \nu} K^{\mu} K^{\nu}
$$

which describes propagation of a light (radio waves) ray from the quasar to the observer (VLBI station). Wave Eq. (3.8) and ray Eq. (3.9) are supplementary to each other and have equivalent physical content.

A more straight-forward solution can be obtained to Eq. (3.8). If Jupiter's acceleration is neglected, a plane-wave solution of this equation is

$$
\varphi\left(x^{\alpha}\right)=\varphi_{0}+k_{\alpha} x^{\alpha}+\frac{2 G M_{J}}{c^{2}}\left(k_{\alpha} u^{\alpha}\right) \ln \left(-k_{\alpha} r^{\alpha}\right)
$$

where $\varphi_{0}$ is a constant of integration, $k^{\alpha}=c^{-1}(\nu, \nu \boldsymbol{k})$ is a constant wave vector of the unperturbed radio wave such that $\eta_{\mu \nu} k^{\mu} k^{\nu}=0, \nu$ is its unperturbed constant frequency. The speed $c$ in the expression for the wave vector $k^{\alpha}$ must be conceptually understood as the speed of light, and the unit vector $\boldsymbol{k}$ defines the unperturbed direction of propagation of the radio wave from the quasar. One can easily prove that Eq. (3.10) is a particular solution of the electromagnetic eikonal Eq. (3.8). Indeed, observing that

$$
\partial_{\alpha} r^{\mu}=\delta_{\alpha}^{\mu}-\frac{u^{\mu}}{\gamma} \partial_{\alpha} s
$$

where $\gamma=\left(1-v_{J}^{2} / c^{2}\right)^{-1 / 2}$, one obtains from the gravity null cone Eq. 2.6

$$
\partial_{\alpha} s=-\gamma \frac{r_{\alpha}}{r_{R}}
$$

Differentiation of Eq. (3.10) using Eqs. (3.11) and (3.12) shows that the eikonal Eq. (3.8) is satisfied.

Eq. (3.10) for the electromagnetic phase is clearly Lorentz-invariant and valid in an arbitrary coordinate system. The gravity field (transverse-traceless and gauge modes) propagates from Jupiter along the hypersurface of gravity null cone (2.7) and perturbs the phase front of the radio wave at a field point $x^{\alpha}$ (see figure 11). Notice that the procedure of solving of the eikonal Eq. (3.8) does not change the physical meaning of the retarded time $s$ in the coordinate of Jupiter $x_{J}^{\alpha}(s)$, affected by the propagation of the gravity. Eq. (2.7) does not depend on the wave vector $k^{\alpha}$ which alone contains the information about the speed and direction of propagation of the quasar's radio wave. In other words, a gravitating body (Jupiter) always perturbs the electromagnetic phase and deflects the light by acting from its retarded position $\boldsymbol{x}_{J}(s)$ regardless of the direction of motion of the incoming photon and/or the magnitude of its impact parameter with respect to the light-ray deflecting body. Hence, the speed of the physical light (radio waves) used for VLBI observations can not enter in any way to the gravity null cone Eq. (2.7). The light is used as a test particle in order to measure the retardation of gravity effect through the retarded position of Jupiter $\boldsymbol{x}_{J}(s)$ which is determined in the experiment from the magnitude and direction of the gravitational deflection of light and is compared with the JPL ephemeris position of Jupiter obtained independently from its direct observation in a series of radio-tracking and/or VLBI measurements of spacecrafts which orbited Jupiter 27] (see Fig. B] for comparison of the two positions of Jupiter).

This independence of the retarded time $s$ from the electromagnetic wave vector $k^{\alpha}$ clearly shows that the retardation is not due to the propagation of the radio wave from the quasar. The radio propagation can not be responsible for the retardation effect in Jupiter's position because the radio wave propagates in a different direction (see Fig. 2). The retardation in Jupiter's position is a consequence of the finite value of the fundamental speed of propagation of gravity and exists independently of solving the eikonal equation; hence the solution of the eikonal equation can not change this property of the propagating gravity field. This conclusion is supported in more detail in [21, 28, 29, 230].

\subsection{The gravitational time delay}

The Lorentz-invariant relativistic time delay equation, generalizing the static Shapiro delay [32], can be obtained directly from Eq. (3.10). We note that the phase $\varphi$ of electromagnetic wave, emitted at the point $x_{0}^{\alpha}=\left(c t_{0}, \boldsymbol{x}_{0}\right)$ and received at the point $x^{\alpha}=(c t, \boldsymbol{x})$, remains constant along the wave's path [3, 25, 31]. Indeed, if $\lambda$ is an affine parameter along the path, one has for the phase's derivative

$$
\frac{d \varphi}{d \lambda}=\frac{\partial \varphi}{\partial x^{\alpha}} \frac{d x^{\alpha}}{d \lambda}=K_{\alpha} K^{\alpha}=0
$$


which means that $\varphi\left(x^{\alpha}(\lambda)\right)=$ const in accordance with our assertion. Equating two values of the phase at the points $x_{0}^{\alpha}$ and $x^{\alpha}$ and separating time and space coordinates one obtains from (3.10)

$$
t-t_{0}=\frac{1}{c} \boldsymbol{k} \cdot\left(\boldsymbol{x}-\boldsymbol{x}_{0}\right)-\frac{2 G M_{J}}{c^{3}} \frac{1-c^{-1} \boldsymbol{k} \cdot \boldsymbol{v}_{J}}{\sqrt{1-v_{J}^{2} / c^{2}}} \ln (r-\boldsymbol{k} \cdot \boldsymbol{r})+\text { const },
$$

where 'const' denotes all of the constant terms and the relativistic terms taken at the emitting point, the distance $\boldsymbol{r}=\boldsymbol{x}-\boldsymbol{x}_{J}(s), r=\left|\boldsymbol{x}-\boldsymbol{x}_{J}(s)\right|$, and the retarded time $s$ is defined by the gravity cone Eq. (2.7). The Lorentz-invariant expression for time delay (3.14) was derived first by Kopeikin and Schäfer [16] who solved equations for light geodesics in the gravitational field of moving bodies. Klioner [33] also obtained this expression by making use of the Lorentz transformation of Jupiter's coordinate and the light vector in the Shapiro delay (which is implicitly equivalent to transforming the gravity and electromagnetic field equations) from a static to moving frame. Notice that in GR Eq. (3.14) describes a hypersurface of both the light and gravity null cones along which electromagnetic field from the quasar and the gravitational field from Jupiter are propagating respectively (see Fig. 1). The light cone intersects with the gravity null cone given by Eq. (2.7) at the point $x^{\alpha}$ at which the propagating Jupiter's gravity field reaches the hypersurface of electromagnetic phase at time $t$ when Jupiter is located at its retarded position $\boldsymbol{x}_{J}(s)$ coinciding with the origin of the retarded gravity null cone. Additional details are shown in appendix where we used a bi-metric theory of gravity. In this theory the light null-cone does not coincide with the gravity null-cone as it was in GR, and it implies that light can not propagate on the hypersurface of the gravity null cone (2.7). Hence, the retarded position of Jupiter measured in Jovian experiment is due to the finite value of the fundamental speed of gravity and it sets the upper limit on the speed of propagation of gravitational waves which can propagate either on shell of the gravity null-cone (2.7) in case of GR [22] or inside it in the case of the theory of gravity with massive gravitons [34].

\subsection{The aberration of gravity}

Let us start with the light geodesics Eq. (3.9). Taking partial derivative from the metric tensor recasts Eq. (3.9) to the following form

$$
\frac{d K_{\alpha}}{d \lambda}=\frac{2 G M_{J}}{c^{2}}\left(k_{\mu} u^{\mu}\right)^{2} \partial_{\alpha}\left(\frac{1}{r_{R}}\right)=-\frac{2 G M_{J}}{c^{2}} \frac{\left(k_{\mu} u^{\mu}\right)^{2}}{r_{R}^{2}} n_{\alpha},
$$

where 15

$$
n^{\alpha} \equiv \partial^{\alpha} r_{R}=\frac{r^{\alpha}}{r_{R}}-u^{\alpha}
$$

and we have neglected Jupiter's acceleration.

The vector $r^{\alpha}$ is null due to the gravity null cone Eq. (2.6), but the vector $n^{\alpha}$ is space-like, because $n_{\alpha} n^{\alpha}=+1$. This occurs because the retardation of gravity effect in the first term in the right side of Eq. (3.16) (present in the coordinate $\boldsymbol{x}_{J}(s)$ of Jupiter through the retarded time $\left.s\right)$ is compensated by the second term depending on the four-velocity of Jupiter $u^{\alpha}$ which is a time-like vector, $u_{\alpha} u^{\alpha}=-1$. This velocity-dependent term present in the gravitational force is associated in GR with the relativistic effect known as aberration of gravity [10] because it describes an aberration-like change in the direction of the gravitational force (that is, from the null-like vector $r^{\alpha} / r_{R}$ to the space-like vector $n^{\alpha}$ ) caused by motion of the gravitating body with respect to the reference frame used for data reduction.

The compensation of the retardation of gravity by the aberration of gravity in the expression for vector $n^{\alpha}$ can be demonstrated using a Taylor (post-Newtonian) expansion of $x_{J}^{\alpha}(s)$ around time $t$. This gives

$$
\begin{aligned}
& r^{\alpha}=R^{\alpha}-(s-t) \frac{d x^{\alpha}}{d s}=R^{\alpha}+\frac{r}{c} \frac{u^{\alpha}}{\gamma}, \\
& r_{R}=-u_{\mu} R^{\mu}+\frac{r}{c \gamma},
\end{aligned}
$$

where terms depending on Jupiter's acceleration have been neglected, $R^{\alpha}=x^{\alpha}-x_{J}^{\alpha}(t)=\left(0, \boldsymbol{x}-\boldsymbol{x}_{J}(t)\right)$ is a purely spatial vector lying on the hypersurface of constant time $t, r=\left|\boldsymbol{x}-\boldsymbol{x}_{J}(s)\right|$, and the retarded time Eq. (2.7) has replaced $s-t$ with $r / c$ where $c$ in this case is understood as the fundamental speed of gravity. Substitution of Eqs. (3.17), (3.18) into Eq. (3.16), and reduction of similar terms, show that retarded term proportional to $s-t$ cancels 
with the gravity aberration term proportional to $u^{\alpha}$ and Eqs. (3.16) and (3.18) are reduced to

$$
\begin{aligned}
& n^{\alpha}=\left[R^{\alpha}+u^{\alpha}\left(u_{\mu} R^{\mu}\right)\right] r_{R}^{-1}, \\
& r_{R}=\left[R_{\alpha} R^{\alpha}+\left(u_{\alpha} R^{\alpha}\right)^{2}\right]^{1 / 2} .
\end{aligned}
$$

This result, shown elsewhere [10], reveals that (in the first approximation with respect to the four-velocity $u^{\alpha}$ ) the gravitational acceleration of a light particle (photon) is directed toward the present position of Jupiter $\boldsymbol{x}_{J}(t)$ separated from the photon by a space-like vector $R^{\alpha}$. At first glance, this might imply that the aberration of gravity cannot be measured in the linear $v_{J} / c$ approximation beyond the Shapiro delay. This point of view is advocated in [5, 6, 7, 8, 9].

However, it is crucial to understand that the VLBI experiment does not measure the gravitational acceleration (the right side of Eq. (3.15)) of radio wave (light particles) which is not VLBI-measurable quantity. What VLBI measures in reality is the electromagnetic phase of the radio wave from a quasar and direction of its propagation, which do depend on the retarded position of Jupiter $\boldsymbol{x}_{J}(s)$ in contrast to the gravitational acceleration. In order to see how it happens let us integrate the inverse distance $1 / r_{R}$ along the light-ray trajectory parametrized by the affine parameter $\lambda$. It results in

$$
\frac{d \lambda}{r_{R}}=-\frac{1}{\gamma} \frac{d s}{k_{\alpha} r^{\alpha}}=\frac{1}{k_{\alpha} u^{\alpha}} d\left[\ln \left(-k_{\alpha} r^{\alpha}\right)\right],
$$

which can be easily confirmed by direct differentiation of the gravity null cone Eq. (2.6) taken on the trajectory of the radio wave signal $x^{\alpha}=x^{\alpha}(\lambda)$. Using Eq. (3.21) for the integration of the light geodesic Eq. (3.15) gives

$$
K_{\alpha}=k_{\alpha}+\frac{2 G M_{J}}{c^{2}} \frac{k_{\mu} u^{\mu}}{k_{\beta} r^{\beta}}\left[k_{\alpha}+\left(k_{\nu} u^{\nu}\right) \frac{r_{\alpha}}{r_{R}}\right],
$$

which coincides with the expression for $K_{\alpha}=\partial_{\alpha} \varphi$, derived by differentiation of the electromagnetic phase (3.10). The subsequent post-Newtonian expansion (3.17) of $r^{\alpha}$ in the function $k_{\alpha} r^{\alpha}$, that enters solution (3.22) for $K_{\alpha}$ and that (3.10) for the phase $\varphi\left(x^{\alpha}\right)$, gives terms which depend on the aberration and the fundamental speed of gravity already in linear order $\sim v_{J} / c$. This result is confirmed in [21, 28] using general relativity parametrized by a single speed-of-gravity parameter $c_{g}$ characterizing all non-stationary effects of gravitational field.

\subsection{Why can VLBI determine the retarded position of Jupiter?}

The gravitational force exerted by moving Jupiter on any mass (including radio photons) is a spatial vector directed in the first approximation toward the present position of Jupiter as shown in Eqs. (3.15) and (3.19). A question arises: "How can the gravitational force be a spatial vector but the phase of electromagnetic waves, measured by VLBI, be gravitationally affected from null direction given by Jupiter's retarded position?" The answer to this question arises from the nature of gravity and radio waves which both move along null rays and their wave fronts form a null hypersurface (null cone).

The gravitational 4-force on the right side of Eq. (3.15) has a Newtonian analog, and for this reason this force is a space-like vector directed to the present position of Jupiter $x_{J}^{\alpha}(t)$. Integration of the 4-force along particle's trajectory gives a vector of a linear momentum of the particle which is tangent to particle's world line. In the case of a slowly moving particle, its linear momentum is a time-like vector. However, radio photons move along light geodesics and their linear momentum is a null wave vector [3, 25]. Hence, the integration of the light geodesics (3.15) along the photon trajectory must produce the null vector $K^{\alpha}=k^{\alpha}+\delta k^{\alpha}$ of the photon, where $k^{\alpha}$ is an unperturbed wave vector of the photon and $\delta k^{\alpha}$, given by Eq. (3.22), is its gravitational perturbation caused by the space-like vector of the gravitational force in the right side of the equations of motion (3.15).

The null vector $K^{\alpha}$ is a gradient of the radio phase with $K^{\alpha}=g^{\alpha \beta} \partial_{\beta} \varphi$ and the phase $\varphi\left(x^{\alpha}\right)$ is a null hypersurface. The gravitational field of Jupiter also propagate along null characteristics defined by vector $r^{\alpha}=x^{\alpha}-x_{J}^{\alpha}(s)$, where $x_{J}^{\alpha}(s)$ is a retarded position of Jupiter taken at the retarded time $s$ defined by the gravity null cone Eq. (2.7). For this reason, the characteristics of the gravitational field of moving Jupiter form a null hypersurface, gravitational phase $\varphi_{g r}\left(x^{\alpha}\right)$, as well. Radio photon interacts with the gravitational force at point $x^{\alpha}$. Therefore, two null hypersurfaces $\varphi\left(x^{\alpha}\right)$ and $\varphi_{g r}\left(x^{\alpha}\right)$ intersect at this point and form a null sub-hypersurface such that both vectors $K^{\alpha}$ and $r^{\alpha}$ belong to it, and the gravitational perturbation of the radio phase, $\varphi\left(x^{\alpha}\right)$, is defined by the structure of this null sub-hypersurface.

There are only three scalars that can be formed from the three vectors $k^{\alpha}, r^{\alpha}$, and $u^{\alpha}$ (Jupiter's velocity): $k_{\alpha} u^{\alpha}$, $r_{R}=-u_{\alpha} r^{\alpha}$, and $k_{\alpha} r^{\alpha}$, and the (weak) perturbation of the radio wave phase can depend on a linear combination of some functions having these three scalars as arguments. The scalar $r_{R}$, however, can be eliminated because the 
partial derivative of the phase $\partial_{\alpha} \varphi$ must be a null vector, whereas the partial derivative $\partial^{\alpha} r_{R}=n^{\alpha}$ (see Eq. (3.16) ) is a space-like vector which does not lie on the null hypersurface. The scalar $k_{\alpha} u^{\alpha}$ is clearly a constant, if one neglects Jupiter's acceleration, so that $\partial^{\beta}\left(k_{\alpha} u^{\alpha}\right)=0$. Thus, term $k_{\alpha} u^{\alpha}$ has no influence on the propagation direction of the photons. We conclude that the only significant scalar combination defining the gravitational perturbation of the electromagnetic phase $\varphi$ is simply $k_{\alpha} r^{\alpha}$, which depends on the retarded position $x_{J}^{\alpha}(s)$ of Jupiter taken at the retarded instant of time $s$ defined by the gravity null cone Eq. (2.7) obtained as a retarded solution of the gravity field Eqs. (2.3). The exact solution of the eikonal Eq. (3.8) confirms the above argument, and shows explicitly that the radio phase $\varphi$ is sensitive to the retarded position of Jupiter $\boldsymbol{x}_{J}(s)$, whereas the gravitational force, acting on photons as a space-like vector, is sensitive to the present position of Jupiter $\boldsymbol{x}_{J}(t)$.

In summary, the solution of the Einstein equations shows that gravity force propagates from a moving massive body; hence the retarded position of the body, if the speed of gravity propagation is not infinite, is a natural consequence of the theory. But, the interaction of gravity in the dynamics of slowly moving bodies $(v \ll c)$ will not produce a null vector since their four-velocities are not partial derivatives of a null hypersurface, and the retardation of gravity effect is relegated to higher orders of $v / c$ in the solutions of the equations of motion for these bodies [35, 36, 37]. On the other hand, the phase (or time delay) of the radio waves which is measured by VLBI, is sensitive to the retardation of gravity effect (retarded position of Jupiter) at order $v / c$ because in GR gravity has the same speed as light which means that the fundamental speed in the Maxwell and Einstein equations is one and the same. This prediction of general relativity was the goal of experimental testing in Jovian experiment.

\subsection{Fundamental speed of gravity as a fitting parameter}

Our theoretical formulation of the problem of propagation of light in a time-dependent gravitational field of moving bodies predicts that the retarded coordinate of Jupiter $\boldsymbol{x}_{J}(s)$ in all terms depending on gravity field in Eqs. (2.5)-(3.10) is shifted from its present position $\boldsymbol{x}_{J}(t)$ due to the finite time the gravity takes to propagate from a moving massive body (Jupiter) to the field point $x^{\alpha}$. To quantify this prediction experimentally we have developed a co-ordinated fiber-bundle parameterization of both the Einstein equations and light geodesics, based on a (single) speed-of-gravity parameter $c_{g}$. This $c_{g}$-parametrized approach is explained in our papers [21, 28] ${ }^{3}$. This $c_{g}$-parameterization decouples the null characteristics of electromagnetic and gravitational field already at the first order terms in $v / c$ in contrast to the PPN formalism by Nordtvedt and Will 6] which starts to distinguish the light and gravity null cones only in terms of the second order in $v / c$ [28]. We assert that on each bundle of the parametric general-relativistic space-time we are allowed to substitute Eq. (2.7) with the following description of the characteristics of the gravity field equations

$$
s_{g}=t-\frac{1}{c_{g}}\left|\boldsymbol{x}-\boldsymbol{x}_{J}\left(s_{g}\right)\right|,
$$

where $c_{g}$ is a speed of gravity parameter running formally from $c_{g}=\infty$ to $c_{g}=c$. Again, we assert that in the papers [21, 28] we are not using $c_{g} \neq c$ in the sense of an alternative theory of gravity. We work with various values of $c_{g}$ in the fiber-bundle space-times of GR from which only one coincides with physical space-time when $c_{g}=c$. Parameter $c_{g}$ must be considered rather as a label marking matrix of the Lorentz transformation of the gravitational field variables [28], making it clearly different from the matrix of the Lorentz transformation of electromagnetic field which depends on the speed of light $c$. For this reason $c_{g}$ is not a measure of the PPN parameter $\alpha_{1}$ which is associated with preferred frame-velocity tests of GR, described in [20] (see misconception 7).

Values of $c_{g}$ less than $c$ (although such values can be formally obtained from the fitting procedure) are strongly restricted by observations of cosmic rays. Indeed, if $c_{g}$ were significantly less than $c$, the cosmic rays would radiate energy mostly in the form of gravitational Cherenkov's radiation, which contradicts the observations [38]. Eq. [3.23) is valid up to the post-Newtonian terms of second order in the sense that it is obtained as a retarded solution of the Einstein equations where all time derivatives contain a single speed-of-gravity parameter $c_{g}$ [21, 28]. The value of the parameter $c_{g}$ in our model of the experiment was found by minimizing the phase residuals $\delta \varphi=\left|\varphi_{\text {obs }}-\varphi_{\text {cal }}\left(x^{\alpha}\right)\right|$, where $\varphi_{\text {obs }}$ is the observed value of the phase, and

$$
\varphi_{\text {cal }}\left(x^{\alpha}\right)=\varphi_{0}+k_{\alpha} x^{\alpha}+\frac{2 G M_{J}}{c^{2}}\left(k_{\alpha} u^{\alpha}\right) \ln \left(-k_{\alpha} \rho^{\alpha}\right)
$$

which is the model function for the phase obtained from Eq. (3.10) after mathematically legitimate replacement of $r^{\alpha}$ with $\rho^{\alpha}=x^{\alpha}-x_{J}^{\alpha}\left(s_{g}\right)[21,28]$.

\footnotetext{
${ }^{3}$ Appendix of the present paper gives another theoretical parametrization of the speed of gravity versus the speed of light
} 
We have introduced the speed-of-gravity parameter $c_{g}$ to Eqs. (3.23) and (3.24) in such a way that eikonal Eq. (3.8) does not change the physical identity of the gravity null cone Eq. (2.7) which treats the retardation as due to the finite speed of the gravity field. The solution to the Einstein equations remains the same as in Eq. (2.5) with $s$ replaced with $s_{g}[21,28]$. This $c_{g}$ speed-of-gravity parameterization of the Einstein equations demands that the speed of the gravitational field is the same in both the wave and near zones of an isolated system emitting gravitational waves. Our experimental goal was to measure $c_{g}$ in the near zone, and to determine if $c_{g}=c$ predicted by general relativity. As we noted previously, the experiment can not measure the speed of gravitational waves in the wave zone directly but the measurement of $c_{g}$ in the near zone definitely imposes the upper limit on the speed of their propagation everywhere.

The retardation of gravity is unobservable in the solutions of the post-Newtonian equations of motion of selfgravitating bodies (a binary pulsar and the Earth-Sun, for example) in terms of order $v / c$ and $v^{3} / c^{3}$ beyond the Newtonian law of gravity because of a perfect cancellation of the retarded and velocity-dependent terms present in the gravitational force between the bodies [35, 36, 37]. However, the present paper (see also [1, 29, 30]) shows that the retarded terms are not canceled in the solution (3.10) of the eikonal equation by the velocity-dependent terms. The phase $\varphi$ has an argument $k_{\alpha} r^{\alpha}$ which does not depend on the velocity of Jupiter explicitly. The post-Newtonian expansion of $k_{\alpha} r^{\alpha}$ around the present time $t$ generates velocity-dependent terms which are physically explained by the finite speed of gravity.

Our $c_{g}$ parametrization [21, 28] assumes that the speed-of-gravity parameter $c_{g}$ appears in the equation for the time delay $t-t_{0}$ taken by light to propagate from $\boldsymbol{x}_{0}$ to $\boldsymbol{x}$ in the following form

$$
t-t_{0}=\frac{c^{3}}{2 \nu^{2}} \int_{t_{0}}^{t} d \zeta \int_{-\infty}^{\zeta} k^{\mu} k^{\nu}\left[k^{i} \frac{\partial h_{\mu \nu}(\tau, \boldsymbol{x})}{\partial x^{i}}+\frac{1}{c_{g}} \frac{\partial h_{\mu \nu}(\tau, \boldsymbol{x})}{\partial \tau}\right]_{\boldsymbol{x}=c \boldsymbol{k}\left(\tau-t_{0}\right)+\boldsymbol{x}_{0}} d \tau
$$

where $k^{\alpha}$ is a wave vector of light, $\nu$ is its frequency, and $\boldsymbol{k}=\left(k^{i}\right)$ is a unit vector along the direction of its unperturbed propagation. Integration of Eq. (3.25) leads exactly to formula (3.14) with the retarded time $s$ replaced with $s_{g}$ defined in Eq. 3.23 of this section. It is clear that in case $c_{g}=\infty$ the Jovian deflection experiment would not measure the integral from the time derivative of the metric tensor shown as the second (gravito-magnetic) term in the right side of Eq. (3.25). However, we have measured $c_{g} \simeq c$ with the precision $20 \%$ and, thus, proved that the experiment is sensitive to the gravito-magnetic dragging of light generated by the partial time derivative of the metric tensor 21, 28].

\subsection{The determination of the speed of gravity}

The speed-of-gravity parameter was determined from the experiment in the following way. Let us introduce two angles $\Theta=\Theta(t)$ and $\theta=\theta\left(s_{g}\right)$. The angle $\Theta$ is between the unit vector $\boldsymbol{k}$ characterizing direction of propagation of the light ray and the unit vector $\boldsymbol{p}=\boldsymbol{R} /|R|$ while the angle $\theta$ is between vector $\boldsymbol{k}$ and $\boldsymbol{l}=\boldsymbol{l}\left(s_{g}\right)=\boldsymbol{\rho} / \rho$. Here $\boldsymbol{R}=\boldsymbol{x}-\boldsymbol{x}_{J}(t)$ connects the present position of Jupiter $\boldsymbol{x}_{J}(t)$ at the time of observation $t$ and the point of observation $\boldsymbol{x}$, whereas $\boldsymbol{\rho}=\boldsymbol{x}-\boldsymbol{x}_{J}\left(s_{g}\right)$ connects the retarded position of Jupiter at the retarded time $s_{g}=t-\rho / c_{g}$ and the point $\boldsymbol{x}$ (see Eq. (3.23) ). By definition $\cos \Theta=\boldsymbol{k} \cdot \boldsymbol{p}$, and $\cos \theta=\boldsymbol{k} \cdot \boldsymbol{l}$ (see figure 2).

From the definitions of vector $\boldsymbol{l}\left(s_{g}\right)$ and the angle $\theta\left(s_{g}\right)$, we can obtain their post-Newtonian expansions in the near zone of the solar system by using Eq. (3.17). Notice that $\boldsymbol{l}(t)=\lim _{s_{g} \rightarrow t} \boldsymbol{l}\left(s_{g}\right) \equiv \boldsymbol{p}$, and $\theta(t)=\lim _{s_{g} \rightarrow t} \theta\left(s_{g}\right) \equiv \Theta$. The argument of the logarithm in Eq. (3.24) for $\varphi_{\text {cal }}$ is

$$
-k_{\alpha} \rho^{\alpha}=\frac{\nu \rho}{c}\left[1-\cos \theta\left(s_{g}\right)\right]
$$

The post-Newtonian expansion of the model function of the electromagnetic phase yields

$$
\varphi_{\text {cal }}=\varphi_{0}+k_{\alpha} x^{\alpha}+\frac{2 G M_{J}}{c^{2}}\left(k_{\alpha} u^{\alpha}\right) \ln \left\{1-\boldsymbol{k} \cdot\left[\boldsymbol{p}+\frac{1}{c_{g}} \boldsymbol{p} \times\left(\boldsymbol{v}_{J} \times \boldsymbol{p}\right)\right]\right\}+O\left(\frac{v_{J}^{2}}{c_{g}^{2}}\right),
$$

where the constant and $\ln \rho$ terms have been neglected because they are smaller than the other relativistic terms, and are much smaller than the experimental angular resolution.

The term in square brackets of the logarithmic function describes a small displacement of vector $\boldsymbol{p}$ to the retarded direction

$$
\boldsymbol{l}=\boldsymbol{p}+\frac{1}{c_{g}} \boldsymbol{p} \times\left(\boldsymbol{v}_{J} \times \boldsymbol{p}\right)
$$


This equation is independent of the electromagnetic wave vector $\boldsymbol{k}$ and describes a change from the present (ephemeris) position of Jupiter given by vector $\boldsymbol{p}$ to its retarded location given by vector $\boldsymbol{l}$ (see figure 21). This displacement is caused by the propagation of gravity force in the near zone of Jupiter with finite speed, and is associated with the near-zone aberration of Jupiter's gravity field.

The determination of $\varphi_{0}$, the unperturbed direction to the quasar $\boldsymbol{k}$, and coordinates of Jupiter in $\varphi_{\text {cal }}$ can be obtained to sufficient accuracy using the JPL ephemeris of the solar system objects with respect to the quasi-inertial reference frame, defined by the International Celestial Reference Frame grid of quasars in the sky [2]. This allows the measurement of the retardation of gravity incorporated in Eq. (2.7) for retarded time $s$.

As we emphasized in previous sections, the aberration of gravity (3.28) is unobservable in the case of the gravitational interaction between two slowly-moving bodies [10]. However, the gravitational perturbation of the electromagnetic phase (not the acceleration) of the radio wave was measured in the experiment in order to extract information about the direction to the position of Jupiter which, in addition to the well-known (radial) Einstein light deflection, has gravitational drag of the radio wave (tangential deflection) by means of the gravito-magnetic force produced by its orbital motion. This gravito-magnetic dragging is equivalent to the aberration of the gravity force.

At the time of the closest approach of Jupiter to the quasar on 2002 September 8 , the angle $\Theta \sim 3.7^{\prime}=10^{-3}$ rad, so that $1-\boldsymbol{k} \cdot \boldsymbol{p}=(1 / 2) \Theta^{2}+O\left(\Theta^{4}\right)$. On the other hand the post-Newtonian parameter $v_{J} / c=4.5 \times 10^{-5} \ll \Theta$. Hence $v_{J} /(c \Theta) \ll 1$, and the aberration of gravity post-Newtonian Eq. (3.27) can be expanded ${ }^{4}$ with respect to a small parameter $v_{J} /(c \Theta)$. It yields equation

$$
\varphi_{\text {cal }}=\varphi_{0}+k_{\alpha} x^{\alpha}+\frac{4 G M_{J}}{c^{2}}\left(k_{\alpha} u^{\alpha}\right)\left[\ln \Theta-\frac{\boldsymbol{k} \cdot \boldsymbol{v}_{J}-(\boldsymbol{k} \cdot \boldsymbol{p})\left(\boldsymbol{p} \cdot \boldsymbol{v}_{J}\right)}{c_{g} \Theta^{2}}\right]+O\left(\frac{v_{J}^{2}}{c_{g}^{2}}\right)
$$

which was used to estimate the magnitude of the aberration of gravity effect [2] described by the second term in square brackets. The magnitude of the aberration of gravity is inversely proportional to the speed-of-gravity parameter $c_{g}$ which has been measured in this experiment as $c_{g}=1.06 \pm 0.21$ in geometrized units 2]. Higher angular accuracy is needed in order to detect TT gravitational waves and measure their speed directly in the wave zone with existing [40] or future [4] technologies of the gravitational-wave detectors and/or other technique [42].

\section{COMMON MISCONCEPTIONS ASSOCIATED WITH THIS EXPERIMENT}

\subsection{Misconception 1: The gravitational field of a uniformly moving body is static}

This misconception is associated with the following reasoning. The gravitational field of a uniformly moving body can be obtained by solving Einstein's equations in the static frame of the body with its subsequent Lorentz transformation to the moving frame. Since the gravitational field in the body's reference frame is static and not propagating, this "non-propagation" property of the gravitational field will be preserved after making the Lorentz transformation to the moving frame (see, for example, [43]).

Any field is called propagating if it is obtained as a solution of the wave-type equation. A field is static if, and only if, it is obtained as a solution of elliptic-type equation. But the linearized Einstein's equations in the moving frame are not of the elliptic-type, they contain time derivatives and are essentially of the wave Eqs. (2.3). The causal (retarded) solution of these equations describes the gravitational field of a uniformly moving body which depends on time. This dependence is represented in the form of a causal gravity wave, Eq. (2.5), propagating from the moving body (Jupiter) to the field point $x^{\alpha}$ along the hypersurface of gravity null cone (2.7). The gravity null cone structure is hidden in the static solution of Einstein's equations in the rest frame of the body. But, a Lorentz transformation from the static frame of the body to a moving frame preserves the structure of the gravity null cone, as Einstein's equations insist, but now makes the cone characteristics measurable by observing the retarded position from which the gravity field of the moving body deflects light (see Figs. 1-3). As discussed previously, the null-cone gravity-field characteristics become measurable in the moving frame because the time derivatives of the Einstein equation are normalized to the fundamental speed (of gravity), and these terms produce the retarded gravitational field, which is not static.

\footnotetext{
${ }^{4}$ Notice that this expansion is valid until the relationship $v / c<\Theta$ is hold. It is incompetent to take the limit $\Theta \rightarrow 0$ for fixed value of $v / c$ as mistakenly done by Asada [39]
} 


\subsection{Misconception 2: The propagation of the gravitational field of a uniformly moving body can not be observed}

The gravitational force acting on a test particle moving in the field of a uniformly-moving massive body is directed in the first approximation to the present position of the body in accordance with Eqs. (3.19) and (3.20). Thus, it is assumed that the propagating property (retardation) of the gravity field of a uniformly moving body cannot be observed [4].

This is true if the trajectory of the test particle is the only measurable quantity. The normal use of relativistic celestial mechanics generally deals with such problems as the motion of binary systems and solar system objects which velocities are much smaller than the fundamental speed of gravity. However, this is not true if the phase of an electromagnetic wave is measured as its front propagates in the field of a uniformly moving body. As GR predicts and this is shown in section 33.4 the phase depends on the retarded position of the body. This retarded position is caused by the finite value of the fundamental speed of gravity in the gravity null cone Eq. (2.7); hence the fundamental speed for gravity can be measured (see appendix for further details).

\subsection{Misconception 3: The parameter $c$ of the Lorentz transformation is physically the speed of light}

The parameter $c$ of the Lorentz transformation is generally associated with the speed of light [5, 6, 8]. However, verbatim interpretation of $c$ as a physical speed of light narrows the true meaning of the Lorentz transformation as describing the transformation property of Maxwell's equations only. Einstein's equations are also wave equations where the wave operator depends on the fundamental constant $c$ which must be interpreted as the speed of propagation of gravitational field. Consequently, the parameter $c$ in the Lorentz transformation associated with the Einstein gravity field equations must be related to the speed of gravity. Generally speaking, parameter $c$ of the Lorentz transformation is just a fundamental constant of the Minkowski space which characterizes an ultimate speed of propagation of any massless field in vacuum. Hence, the name of the "speed of light" for the parameter $c$ in the Lorentz transformation should not be taken literally when one interprets gravitational experiments dealing with non-stationary (propagating) gravitational fields.

\subsection{Misconception 4: The speed of gravity can only be measured through observation of gravitational waves}

The prevailing view is that the speed of gravity can only be associated with the propagation of plane gravitational waves decoupled from the system emitting these waves [43]. Such waves are generated if the second time derivative of the quadrupole moment of the system is not constant [3, 25]; that is, the bodies comprising the system accelerate. These gravitational waves, which are type $\mathrm{N}$ according to Petrov's classification of gravitational fields [17, 26, 44], are indeed generated if, and only if, the bodies accelerate. However, the gravitational field of a system emitting gravitational waves also has contributions from other Petrov's type fields - I, II, and III, which have in general relativity the same structure of the null characteristics as the N-type waves, but decay faster with distance from the system so that these near-zone fields can not be observed as freely propagating waves of type N 17]. Hence, any measurement of the null characteristics of the near-zone gravitational field provides us with the upper limit on the speed of propagation of gravitational waves of type $\mathrm{N}$.

Propagation of light through the multipolar gravitational field of an isolated astronomical system, emitting gravitational waves, in the near, intermediate, and wave zones of a system, have been already studied [18, 45, 46]. We showed that parameters of the light signal (wave vector, polarization, etc.) depends in general on the retarded value of the multipole moments of the system, as well as their first (velocity associated) and second (acceleration associated) time derivatives. The second time derivatives of the multipolar field dominate in the wave zone but plays no significant role in the near and intermediate zones where the non-radiative gravitational fields associated with the retarded value of the multipole moment of the system and its first derivatives prevail. This fields and their propagators can be studied in the light-ray deflection experiments when light moves in the near zone of the isolated system. Thus, a propagating gravitational field should not be associated only with the accelerated motion of the bodies when measurements are made in the near and/or intermediate zone of the system emitting gravitational waves.

In summary, the propagation of gravity is a general concept which must be associated with the wave nature of gravitational field, and is not limited exclusively to plane gravitational waves of Petrov's N type. Thus, a uniformly moving body generates a retarded gravitational field which is associated with velocity of the body. This retarded velocity-field can be also obtained by making Lorentz transformation of the static gravitational field. This transformation induces the aberration of the null characteristics of the gravity field which can be observed using the phase 
of the radio waves and used to measure their speed without direct detection of gravitational waves, as explained in previous sections of this paper.

The velocity-field is well-known in electrodynamics [11, 25, 47] when electromagnetic field of moving charge is calculated in terms of the retarded Liénard-Wiechert electromagnetic potentials. This velocity-field reflects the propagation of the electromagnetic field of a uniformly moving charge but it can be also calculated by making use of the Lorentz transformation. The same is true for gravity.

\subsection{Misconception 5: The retarded position of Jupiter is taken at the time of the light-ray closest approach}

The consensus model used to evaluate the deflection of light by Jupiter (egs. [48]) calculates the time $t^{*}$ of closest approach of the unperturbed light ray to Jupiter, determines the 'retarded' position of Jupiter $\boldsymbol{x}_{J}\left(t^{*}\right)$ at this time, and then calculates the deflection magnitude and direction. This interpretation of the retarded position of the light-ray deflecting body (Jupiter) sounds very plausible but the mathematical solution of the Einstein and eikonal equations reveals that the retarded position of Jupiter must be actually taken at the point $\boldsymbol{x}_{J}(s)$ on Jupiter's world line at the retarded time $s$ defined by the solution of the gravity null cone Eq. (2.7).

General relativity unequivocally predicts that the deflection of the radio photon occurs the most strongly when Jupiter is at its retarded position $\boldsymbol{x}_{J}(s)$ on the gravity null cone (2.7). In the case of a small impact parameter of the light ray to Jupiter, the time of the closest approach $t^{*}$ of the light ray to Jupiter is nearly equal to the retarded time $s$, Eq. (2.7), caused by the finite speed of gravity. Thus, the retarded position $\boldsymbol{x}_{J}(s)$ of Jupiter can be expanded around the time of the closest approach, $\left.\boldsymbol{x}_{J}(s)\right)=\boldsymbol{x}_{J}\left(t^{*}\right)+\left(s-t^{*}\right) \boldsymbol{v}_{J}\left(t^{*}\right)+\ldots$ so that position of Jupiter $\boldsymbol{x}_{J}\left(t^{*}\right)$ taken at the time of the closest approach can be used in calculation of the light-ray deflection with a very good approximation. One should not however confuse the cause and effect - the time $t^{*}$ of the closest approach of the photon to Jupiter is not inherent to the theory at all, but comes from an approximation of small impact parameter of light-ray trajectory to the light-ray deflecting body. It does not mean that the retardation of the gravitational perturbation of light is caused by light propagation. Full treatment of the small and large impact parameter approximations in the case of gravitational lensing is given in $16,18,31,46]$.

\subsection{Misconception 6: Null characteristics of gravity field can be observed only at $(v / c)^{2}$ terms beyond the Shapiro delay}

This misconception arises from the post-Newtonian expansion of the metric tensor perturbation $h_{\alpha \beta}$ in Eq. (2.5) made prior to solving the eikonal Eq. (3.8). Indeed, the post-Newtonian expansion of $h_{\alpha \beta}$, using the Taylor expansion of the retarded variables depending on the retarded time $s$ in Eq. (2.7) around the present time $t$, leads to

$$
h_{\alpha \beta}(s, \boldsymbol{x})=h_{\alpha \beta}(t, \boldsymbol{x})+\text { terms of order }(v / c)^{2},
$$

where $h_{\alpha \beta}(t, \boldsymbol{x})$ are solutions of the elliptic-type equations (Newtonian potentials). Eq. (4.30) suggests that the retardation of gravity effect is postponed to the terms of order $(v / c)^{2}$ because no terms of order $v / c$ is explicitly present in this expansion [6, 7].

The physical reason for the absence of terms of order $v / c$ in Eq. (4.30) is that the Liénard-Wiechert potentials depend on distance $r_{R}=-u_{\alpha} r^{\alpha}$ which incorporates both the retardation and aberration of gravity effects that are equivalent in terms of order $v / c$ and cancel each other when the post-Newtonian expansion of the metric tensor is done. Light propagates in GR with the same speed as gravity. Lorentz-invariant solution of the eikonal equation incorporates the aberration of light which is equal to the aberration of gravity terms and mutually cancel each other, hence, leaving the retardation of gravity terms in the radio wave phase unchanged. That is why the post-Newtonian expansion of the retarded gravitational perturbation of the electromagnetic phase reproduces the aberration of gravity terms which we had observed in the Jovian deflection experiment via the retarded position of Jupiter [2].

Although $h_{\alpha \beta}(t, \boldsymbol{x})$ does not contain terms of order $v / c$, the measurement of these Newtonian-like gravitational potentials by VLBI is indeterminate because the gauge invariance of the Einstein equations make the potentials coordinate-system dependent. There are gauges, like the ADM gauge [3], where $g_{00}$ and $g_{0 i}$ components of the metric tensor become the solution of the elliptic-type equations which suggest that these metric tensor components do not propagate at all. But, of course, any gravitational influence of these potentials on measurable quantities is not instantaneous but propagates with the finite speed. This resembles the situation in electrodynamics where the Coulomb gauge leads to the elliptic-type equation for scalar potential but it does not mean that electric and magnetic fields propagate instantaneously [47]. Hence, the potentials are not directly measurable quantities in the light-ray deflection experiments because they depend on the specific choice of gauge conditions which is a matter of convenience. 
Only those observable quantities which are gauge-independent (like the electromagnetic phase) can be used to probe physical properties of the gravitational fields.

Our method of analysis, given in this paper, consists of two part: first, to solve the problem of the propagation of light in the time-dependent gravitational field by making use of the Liénard-Wiechert potentials; and then to determine the post-Newtonian expansion of the solution in order to produce the physical interpretation in terms of different order in $v / c$. However, we will show below that if the post-Newtonian potentials (4.30) are used to solve the eikonal Eq. (3.8), the solution will contain terms of order $v / c$ which can be represented in several equivalent forms - leading to difficulty in the interpretation of the solution. This difficulty can be resolved if the solution of the eikonal equation at higher orders of $v / c$ is also determined in order to match it smoothly with the linear terms of order $v / c$ present in the phase.

For this experiment, the observable quantity is electromagnetic phase $\varphi$ which is a gauge-independent quantity [3]. We can measure the impact of the gravitational potentials on the electromagnetic phase in the following manner. The post-Newtonian expansion of the electromagnetic phase is

$$
\varphi\left(x^{\alpha}\right)=\varphi_{0}+k_{\alpha} x^{\alpha}+\frac{1}{c^{2}} \psi\left(x^{\alpha}\right),
$$

where $\varphi_{0}$ is constant and $k^{\alpha}$ is the wave vector of electromagnetic phase. Substitution of Eq. 4.31 to the eikonal equation and making use of expansion (4.30) yields the linearized equation for the phase perturbation $\psi$

$$
\frac{1}{c} \frac{\partial \psi}{\partial t}+k^{i} \frac{\partial \psi}{\partial x^{i}}=2\left(1-\frac{\boldsymbol{k} \cdot \boldsymbol{v}_{J}}{c}\right) U_{J}(t, \boldsymbol{x})+O\left(\frac{v_{J}^{2}}{c^{2}}\right),
$$

where $U_{J}(t, \boldsymbol{x})=G M_{J} / R, R=\left|\boldsymbol{x}-\boldsymbol{x}_{J}(t)\right|$, and $k^{i}$ is the unit vector in the direction of propagation of light rays which are orthogonal to the hypersurface of the phase $\varphi$. Eq. (4.32) is a partial differential equation of the first order and its solution is

$$
\psi\left(x^{\alpha}\right)=2 G M_{J}\left(1-\frac{\boldsymbol{k} \cdot \boldsymbol{v}_{J}}{c}\right) \ln [p R-\boldsymbol{p} \cdot \boldsymbol{R}]+O\left(\frac{v_{J}^{2}}{c^{2}}\right)
$$

where $\boldsymbol{p}=\boldsymbol{k}-c^{-1} \boldsymbol{v}_{J}$, and $\boldsymbol{v}_{J}=d \boldsymbol{x}_{J}(t) / d t$ is Jupiter's velocity.

The argument of the logarithmic function in Eq. 4.33) can be represented in two different forms. The first form is given by $[\underline{6}, 24]$

$$
p R-\boldsymbol{p} \cdot \boldsymbol{R}=\left(1-\frac{1}{c} \boldsymbol{k} \cdot \boldsymbol{v}\right)(R-\mathcal{K} \cdot \boldsymbol{R})
$$

where

$$
\mathcal{K} \equiv \frac{\boldsymbol{p}}{p}=\boldsymbol{k}-\frac{1}{c}\left(\boldsymbol{k} \times\left(\boldsymbol{v}_{J} \times \boldsymbol{k}\right)\right)
$$

The second form is

$$
p R-\boldsymbol{p} \cdot \boldsymbol{R}=r-\boldsymbol{k} \cdot \boldsymbol{r}=-k_{\alpha} r^{\alpha},
$$

where $k^{\alpha}$ is a wave vector of the radio wave, $r^{\alpha}=(r, \boldsymbol{r}), \boldsymbol{r}=\boldsymbol{x}-\boldsymbol{x}_{J}(s), r=|\boldsymbol{r}(s)|$, and the retarded time $s$ is given by the gravity null cone Eq. (2.7).

Eq. (4.35) looks similar in form to the aberration of light formula transforming the direction of propagation of the light ray $\boldsymbol{k}$ from the moving frame of an observer to the direction $\mathcal{K}$ in the static frame of Jupiter. This is why Will [ [] and Carlip [7] interpreted the effect we have observed as the aberration of light (see next section and appendix). However, Eq. (4.36) clearly shows that this aberration term should be interpreted as an aberration of gravity caused by its propagation because Jupiter's coordinate depend on the retarded time $s$ defined through the solution of the Einstein (wave) Eq. (2.3). Any aberration, of course, is of order $v / c$ but only one type of aberration of either electromagnetic or gravitational field can be unambiguously extended to terms of second order in $v / c$ to keep Lorentz-invariance of the equations. Our calculations show that aberration term in Eq. (4.35) matches smoothly with the retardation of gravity effect (4.36) in any post-Newtonian order while the aberration of light does not (see also [33] for more detail on the matching procedure). 


\subsection{Misconception 7: The Jovian experiment and the PPN parameter $\alpha_{1}$.}

The Jovian deflection experiment measures the aberration of gravity and its fundamental speed limit. It tests for the first time the non-stationary gravito-magnetic property of the gravitational field, of order $(v / c)^{3}$, generated by the time derivative of the metric tensor (gravito-magnetic displacement current). The experiment can also be viewed as a test of the Lorentz-invariance of the gravitational field and confirmation of its relativistic causal nature.

Will [20] introduced the "preferred frame" PPN parameter $\alpha_{1}$ which characterizes the violation of the Lorentzinvariance of the PPN metric tensor. However, this parameter presents only in $g_{0 i}$ component of the metric tensor if it is written down in the preferred frame. Hence, parameter $\alpha_{1}$ characterizes, in fact, a strength of the physical coupling between the $g_{0 i}$ component of the metric tensor and the matter current given by $T_{0 i}$ component of the stress-energy tensor. Subsequent Lorentz transformation to a frame moving with respect to the preferred frame with velocity $\vec{w}$ introduces a product $\alpha_{1} \vec{w}$ to $g_{00}$ and $g_{0 i}$ components of the metric tensor.

Under the assumption that the cosmic microwave background radiation (CMBR) defines the preferred frame, several accurate experimental tests give $\alpha_{1} \leq 4 \times 10^{-4}$, which appears more accurate than our limit on $\left(c_{g} / c\right)-1 \leq 0.27$, both of which are measures of the experimental departure from GR. However, direct comparison between these two methods for testing Lorentz-invariance is not valid. First, the PPN formulation states that $\alpha_{1}$ is not coupled with time derivatives of the metric tensor whereas the deflection experiment is a direct test of the magnitude of the time derivative of the metric tensor (see Eq. (3.25) and 21, 28]). In GR the parameter $\alpha_{1} \equiv 0$. Therefore, search for $\alpha_{1}$ is an attempt to find an effect, which probably does not exist at all because GR is a valid theory, while our measurement of the fundamental speed of gravity discovers a real (and newly measured) physical effect - the aberration of gravity, which is a fundamental property of gravity predicting that electromagnetic signals are deflected by gravitational field of a moving gravitational body from its retarded position with respect to observer.

Secondly, the current limit on the parameter $\alpha_{1}$ strongly depends on our guess about the velocity $\vec{w}$ of the solar system relative to a "preferred frame" which was assumed to be the CMBR. But, we do not know if it defines the preferred coordinate system in the sense forbidden by special relativity; it is just a convenient frame to describe the global isotropy of the cosmological black-body radiation. Furthermore, future observations of the relic gravitational wave background (GWB), for example, may lead to the frame somehow moving with respect to the CMBR frame. Since the GWB was formed in the very early universe, long before the CMBR decoupled from matter, it would be more likely to associate it with the preferred frame. However, neither the global topological isotropy of CMBR nor GWB may be related to the local Lorentz-invariant isotropy of the space-time itself. Finally, modern multi-connected cosmologies challenge the cosmological Copernican Principle, and indicate that various possibilities for the global preferred frame may not coincide with the CMBR and GWB frames [49]. Thus, the limit on $\alpha_{1}$, established by [20] depends on numerous assumptions about properties of a preferred reference frame and other cosmological principles which are to some extent ambiguous concepts. It seems to us that only GP-B experiment will be able to set up a limit on $\alpha_{1}$ parameter which will not be sensitive to the choice of the preferred frame [50].

\section{OTHER INTERPRETATIONS}

Four alternative interpretations, which disagree with our interpretation and among themselves, of the Jovian deflection experiment have been proposed (see section 11). We have analyzed fully the alternative interpretations elsewhere [21, 28, 29, 30], but summarize the main arguments here.

The main reason for the disagreements between the four other interpretations is their use of the truncated linearized expansion of the Einstein equations which is not capable to study the Lorentz-invariance of the gravity field force in the light-ray deflection experiments in higher orders in $v / c$. The truncated linearized theory is not sufficient to give the proper geometrical picture of the observed quantity in the time delay of light which is, in fact, a Minkowskian dot product $\Phi=-k_{\alpha} r^{\alpha}$ between two null vectors $k^{\alpha}$ and $r^{\alpha}$ (see figure 1) entering the gravitational perturbation of the electromagnetic phase $\varphi$ in Eq. (3.10). When Einstein's theory of the gravitational time delay is truncated at linear $v / c$ order terms, the simple geometric picture of the experiment is easily missed and the interpretation of the experiment becomes obscured.

Will [6] (see also [] ) introduced a set of alternative theories of gravity with a propagation speed $c_{g}$ which could differ from the speed of light $c$. However, the use of the Post-Newtonian Parameterized (PPN) approximation, which is wide-spread, does not keep the geometrical relationship between the Christoffel symbols (light geodesic equations) and Ricci tensor for different values of $c_{g}$. Moreover, the PPN formalism transforms the Christoffel symbols from one inertial frame to another with the matrix of the Lorentz transformation for electromagnetic field 28]. This makes no sense since the Christoffel symbols are defined purely in terms of gravitational potentials which must be transformed in accordance with the group of the Lorentz transformation of gravitational field depending on the parameter $c_{g}$. In addition, the PPN gravity field equations with $c_{g}$ are not derivable from a Lagrangian of any known theory of gravity. 
Hence, they do not provide us with a complete physical description of the gravitational field in case when the speed of gravity $c_{g} \neq c$ making the PPN approach unreliable for analysis of experiments in time-dependent gravitational fields. This may explain why Will's use of the PPN approximation to analyze relativistic corrections to the Shapiro time delay confuses the aberration of light and the aberration of gravity (see appendix for more detail).

As discussed in the previous section, Will's formulation does predict an 'aberration' deflection of order $v / c$, Eq. (4.35), although Will attributes this aberration to propagation of "light". The difference between his interpretation with ours can be resolved by finding explicit terms of the second order in $v_{J} / c$ in the solution of eikonal Eq. (3.8), and then matching them smoothly with the solution (4.33). This matching shows 21] that the correct interpretation of the retarded position of Jupiter in the gravitational perturbation of electromagnetic phase in Eq. (4.33) must be associated with the null characteristics of gravity field. In other words, had the exact aberration-of-light formula been used to account for the second order terms in Eq. (4.35), it would not produce all of the second and higher order terms in the solution of the eikonal Eq. (3.8), whereas the retarded time Eq. (4.36) gives all terms of second and higher orders in the Lorentz covariant solution of the eikonal Eq. (3.8) as shown by the calculations given in the present paper and in [28, 30, 33]. Thus, the correct meaning of the linearized Eq. (4.35) should not be associated with the aberration of light but with the retardation (aberration) of gravity caused by the orbital motion of Jupiter 24] and associated with the finite value of the fundamental speed of gravity ${ }^{5}$.

Asada [5] suggested that the origin of the retarded time Eq. (2.7) is associated with the propagation of the radio waves from the quasar in the field of static Jupiter. However, this equation originates from the Liénard-Wiechert solution of the Einstein equations (2.3), which describes the propagation of gravitational field and is not associated in any way with the propagation of radio waves from the quasar. Propagation of radio waves is described by the time delay Eq. (3.14) which describes the light cone, while the retarded time Eq. (2.7) defines characteristics of the gravity field null cone (see figure 1). Calculations displayed in the appendix of this paper obviously demonstrate that Asada's interpretation [5] is illicit since it confuses the null characteristics of light and gravity.

A fundamental flaw in Samuel's [8] interpretation was his assumption that the direction to Jupiter was directly measured by VLBI network in the deflection experiment so he also confused the null directions associated with characteristics of gravity and radio waves [29]. Even at the minimal separation of 3.7' of Jupiter and the quasar on 2002 September 8, no radio emission from Jupiter was obtained with the VLBI observations. The null direction to retarded position of Jupiter, depending on the value of the fundamental speed of gravity, was measured from the gravitational time delay Eq. (3.14) and compared with that known extremely accurately from the JPL solar system ephemeris depending on the speed of light (see appendix and Fig. 3). Therefore, minimization of the residual electromagnetic phase with the speed of gravity taken as a fitting parameter allowed us to measure it with respect to the speed of light.

Pascual-Sánchez [9] stated that the retarded position of Jupiter measured in the experiment is the Rømer delay of light coming from Jupiter to observer and that this Rømer delay has nothing to do with the gravitational time delay of light. Eq. (2.7) for retarded time $s$ looks indeed similar to the Rømer delay but this delay is for propagation of the gravitational field from moving Jupiter to the observer at Earth as independently proved in the appendix of the present paper and in [28]. It does not describe the Rømer delay of light but gravity which takes finite time to propagate from moving Jupiter to observer. Had the Rømer delay of gravity been different from that for light, Jupiter would deflect light not from its retarded but from some other position on its orbit. The experiment disproved this assumption confirming that the Rømer delay of gravity and light are equal with accuracy of $20 \%$ by comparing two positions of Jupiter on its orbit obtained independently from the gravitational deflection of quasar's light and JPL ephemeris.

\section{SUMMARY}

We have developed a Lorentz-invariant general-relativistic theory of light-deflection experiments associated with moving bodies in the solar system. The formulation is based on the retarded Liénard-Wiechert solution of the Einstein equations, and explicitly calculates the effect on the phase of the radio wave from the quasar caused by the finite speed of gravity which is a primary fundamental constant in Einstein's field equations. Our post-Newtonian expansion of the retarded coordinate of the light-ray deflecting body shows that the retardation of gravity effect after making its postNewtonian expansion, is present already in linear aberration-like terms of order $v / c$ beyond the Shapiro delay; hence, we interpret the $v / c$ term as caused by the aberration of gravity in the expression for the gravitational force (3.15)

${ }^{5}$ AS we mentioned the effect we have observed can be also explained in terms of the gravito-magnetic dragging of the quasar light ray $[21,28]$ 
exerted by Jupiter on the photons. We have used the concept of the gravito-magnetic field caused by translational motion of Jupiter as an aid in understanding the gravitational phenomena associated with this experiment.

The VLBI measurements confirmed that the deflection is associated with the retarded position of Jupiter, with the speed of gravity equal to that of light to $20 \%$ accuracy [2] and this retardation is not a preferred frame effect associated with the PPN parameter $\alpha_{1}$. Other contradictory interpretations of the experimental results are associated with: (1) an analysis of the Einstein equations which are not fully Lorentz-invariant; (2) misconceptions about the experimental method and analysis; (3) misunderstanding of the nature of the interaction of the gravitational field from moving objects with the phase of the quasar radio signal which is a null hypersurface. According to GR the phase is influenced in all orders of $v / c$ by the propagating gravitational field of Jupiter and this perturbation reveals itself in the linearized order of $v / c$ as the aberration of gravity force of Jupiter due to the finite speed of gravity.

We thank the Eppley Foundation for Research (award 002672) for support.

\section{APPENDIX A: PROPAGATION OF LIGHT IN A BI-METRIC THEORY}

In this section we assume that the Einstein theory of general relativity is valid with the time coordinate $x^{0}=c t$ where $c$ denotes the fundamental speed of gravity. Let us assume that light propagates in vacuum with the speed $c_{l}$ less than the speed of gravity $c$. Effectively, this means that vacuum in the presence of the gravity field can be considered as a fully transparent medium which has a constant refractive index $\epsilon \geq 1$. Propagation of light in such vacuum can be treated on the basis of the Maxwell electrodynamics in medium. The geometric optics limit of these equations has been elaborated by Synge [51] and we employ his approach.

According to Synge [51] light propagates in the medium along light geodesics of an optical metric defined by

$$
\begin{aligned}
& \bar{g}_{\alpha \beta}=g_{\alpha \beta}+\left(1-\frac{1}{\epsilon^{2}}\right) V_{\alpha} V_{\beta}, \\
& \bar{g}^{\alpha \beta}=g^{\alpha \beta}-\left(\epsilon^{2}-1\right) V^{\alpha} V^{\beta},
\end{aligned}
$$

where $g_{\alpha \beta}$ is the gravitational metric given by Eqs. 2.1), 2.5), and $V^{\alpha}$ is a vector field associated with four-velocity of the medium. In our case it means that if the speed of gravity and light are not equal there must exist a preferred frame in which the speed of light is isotropic while in all other frames it is anisotropic. Notice that in the preferred frame $V^{\alpha}=(1,0,0,0)$ and the speed of light $c_{l}=c / \epsilon<c$.

There are other bi-metric theories of gravity (see, for example, [7, [52]) with two metrics predicting different speeds of propagation for light and gravity. In such theories the four-velocity $V^{\alpha}$ is identified with a long-range vector field spontaneously violating the Lorentz-invariance of gravity. Those bi-metric theories lead to the geodesic equations of light also propagating along the null cone of the optical metric [7].

Let us assume for simplicity that both the refractive index $\epsilon$ and the vector field $V^{\alpha}$ are constant ${ }^{6}$. This model is simple but self-consistent and sufficient to apparently distinguish the speed of light $c_{l}$ from the speed of gravity $c$. Equations of our bi-metric model and their solution are given in the rest of this appendix.

Light rays in Synge's approach [51] are defined by a covariant equation for electromagnetic phase (eikonal) $\varphi$ which reads ${ }^{7}$

$$
\bar{g}^{\mu \nu} \partial_{\mu} \varphi \partial_{\nu} \varphi=0
$$

which generalizes Eq. (3.8) for propagation of light in the case of $c_{l} \neq c$. Assuming that unperturbed solution of Eq. A.3) is a plane wave we can write a general solution of this equations as follows

$$
\varphi\left(x^{\alpha}\right)=\varphi_{0}+k_{\alpha} x^{\alpha}+\psi\left(x^{\alpha}\right),
$$

where $k_{\alpha}$ is an unperturbed (constant) wave co-vector of the electromagnetic wave, and $\psi(x)$ is a relativistic perturbation of the eikonal generated by the metric tensor perturbation $h_{\alpha \beta}$ defined in Eq. (2.5). Substitution of Eq. (A.4) to A.3 yields

$$
\begin{aligned}
\bar{\eta}^{\alpha \beta} k_{\alpha} k_{\beta} & =0 \\
\bar{\eta}^{\alpha \beta} k_{\alpha} \frac{\partial \psi}{\partial x^{\beta}} & =\frac{1}{2} h^{\alpha \beta} k_{\alpha} k_{\beta},
\end{aligned}
$$

\footnotetext{
${ }^{6}$ More general case can be solved as well but it significantly complicates solution of equations and will be omitted

7 Lagrangian-based approach of an alternative bi-metric theory of gravity [7] leads to the same equation.
} 
where the unperturbed part of the optical metric A.1 , A.2 is defined by

$$
\begin{aligned}
& \bar{\eta}^{\alpha \beta}=\eta^{\alpha \beta}-\left(\epsilon^{2}-1\right) V^{\alpha} V^{\beta}, \\
& \bar{\eta}_{\alpha \beta}=\eta_{\alpha \beta}+\left(1-\frac{1}{\epsilon^{2}}\right) V_{\alpha} V_{\beta} .
\end{aligned}
$$

Let us introduce a light-ray vector $\sigma^{\alpha}$ defining direction of propagation of the unperturbed light ray. One has [51]

$$
\sigma^{\alpha}=\bar{\eta}^{\alpha \beta} k_{\beta}=k^{\alpha}-\left(\epsilon^{2}-1\right)\left(V^{\beta} k_{\beta}\right) V^{\alpha},
$$

which implies that

$$
\bar{\eta}_{\alpha \beta} \sigma^{\alpha} \sigma^{\beta}=0, \quad \text { and } \quad k_{\alpha} \sigma^{\alpha}=0 .
$$

Vector $\sigma^{\alpha}$ points to the direction of propagation of a light ray from a radio source (quasar) to observer (see Fig. 33). Making use of vector $\sigma^{\alpha}$ simplifies Eq. (A.6) and reduces it to the following form

$$
\sigma^{\alpha} \frac{\partial \psi}{\partial x^{\alpha}}=\frac{1}{2} h_{\alpha \beta} \sigma^{\alpha} \sigma^{\beta} .
$$

The unperturbed characteristics of the eikonal Eq. (A.11) are straight lines (light rays) parametrized by the affine parameter $\lambda$ in such a way that

$$
\frac{d}{d \lambda}=\sigma^{\alpha} \frac{\partial}{\partial x^{\alpha}}
$$

Integration of Eq. A.12 by making use of the unperturbed characteristics is straightforward (see, for example, [53]) and is given as follows

$$
\psi\left(x^{\alpha}\right)=-\frac{2 G M}{c^{2}} \frac{\left(\sigma_{\alpha} u^{\alpha}\right)^{2}+(1 / 2)\left(\sigma_{\alpha} \sigma^{\alpha}\right)}{\left(P_{\alpha \beta} \sigma^{\alpha} \sigma^{\beta}\right)^{1 / 2}} \ln \left(-l_{\alpha} r^{\alpha}\right),
$$

where we have defined

$$
\begin{aligned}
l^{\alpha} & =\sigma_{\perp}^{\alpha}+\sigma_{\perp} u^{\alpha} \\
\sigma_{\perp}^{\alpha} & =P_{\beta}^{\alpha} \sigma^{\beta} \\
\sigma_{\perp} & =\left(\sigma_{\perp \alpha} \sigma_{\perp}^{\alpha}\right)^{1 / 2}=\left(P_{\alpha \beta} \sigma^{\alpha} \sigma^{\beta}\right)^{1 / 2}
\end{aligned}
$$

and

$$
P_{\alpha \beta}=\eta_{\alpha \beta}+u_{\alpha} u_{\beta},
$$

is the operator of projection on the hyperplane orthogonal to the four-velocity $u^{\alpha}$ of the light-ray deflecting body so that it obeys to equation $P_{\alpha \beta} P_{\gamma}^{\beta}=P_{\alpha \gamma}$. It is easy to confirm that Eq. A.13 is solution of Eq. A.11 by observing that

$$
\frac{d}{d \lambda} \ln \left(-l_{\alpha} r^{\alpha}\right)=-\frac{\sigma_{\perp}}{r_{R}}
$$

where $r_{R}=-u_{\alpha} r^{\alpha}$, and Eqs. (3.11), (3.12) have been used. Eq. (A.14) allows us to recast the argument of the logarithm in Eq. A.13 as

$$
l_{\alpha} r^{\alpha}=\sigma_{\perp}^{\alpha} r_{\alpha}-\sigma_{\perp} r_{R} .
$$

It is worth noticing that both vectors $l^{\alpha}$ and $r^{\alpha}$ are null vectors of the unperturbed gravity metric $\eta_{\alpha \beta}$, that is,

$$
\begin{aligned}
\eta_{\alpha \beta} l^{\alpha} l^{\beta} & =0, \\
\eta_{\alpha \beta} r^{\alpha} r^{\beta} & =0,
\end{aligned}
$$

which are consequences of definitions given by Eqs. (A.14) and (2.6). Thus, neither $l^{\alpha}$ nor $r^{\alpha}$ belong to the null cone of the optical metric $\bar{g}_{\alpha \beta}$ (see Fig. 3). This point has not been understood by researchers whose interpretations of the 
Jovian deflection experiment differ between themselves. In particular, Asada [5] insisted that the retarded position of Jupiter is on the null cone of the optical metric. Our calculations show that this Asada's assertion is erroneous.

Solution A.4 A.13 for the electromagnetic eikonal in the bi-metric theory should be compared with a similar solution (3.10) for the case of propagation of light in general relativity where gravity and light have the same null cone. The reader can see that the null directions (2.7) of the gravity metric $g_{\alpha \beta}$ enters explicitly the gravitationally perturbed part of the eikonal A.13 in the bi-metric theory in the form of a dot product $l_{\alpha} r^{\alpha}$ in the argument of the logarithm, where $r^{\alpha}$ is the null distance of the metric $g_{\alpha \beta}$ between observer and the light-ray deflecting body ${ }^{8}$. This term replaces the argument $k_{\alpha} r^{\alpha}$ of the logarithmic term in the eikonal (3.10) in general relativity where $c_{l}=c$. A remarkable fact is that both $l^{\alpha}$ and $r^{\alpha}$ are null vectors of the metric $g_{\alpha \beta}$ describing propagation of gravity. Consequently, gravitational light-ray deflection experiments in the field of moving bodies are sensitive to, and can measure, the null characteristics of the gravity metric $g_{\alpha \beta}$ by observing propagation of light that moves along the null characteristics of the optical metric $\bar{g}_{\alpha \beta}$. This fully agrees with our general relativistic interpretation of the Jovian experiment [1, 2].

Analysis given in this appendix provides a firm support to our statement [1, 2] that the retarded coordinate of Jupiter $x_{J}^{\alpha}(s)$ measured in the Jovian experiment is associated with the finite speed of gravity but not light. The statement that the Jovian experiment measures the light-cone effects associated with the optical metric $\bar{g}_{\alpha \beta}$ as claimed in [5, 6, 7, 8, 9, 54] is not valid.

\footnotetext{
8 The amplitude of the eikonal also changes leading to the deviation of the PPN parameter $\gamma$ from its general relativistic value $\gamma=1$.
} 
[1] S. M. Kopeikin, Astrophys. J. Lett. 556, L1 (2001)

[2] E. B. Fomalont and S. M. Kopeikin, Astrophys. J. 598, 704 (2003)

[3] C. W. Misner, K. S. Thorne and J. A. Wheeler, Gravitation (Freeman, New York, 1973)

[4] S. Frittelli, Mon. Not. R. Astron. Soc. 344, L85 (2003)

[5] H. Asada, Astrophys. J. Lett. 574, L69 (2002)

[6] C. M. Will, Astrophys. J. 590, 683 (2003)

[7] S. Carlip, Clas. Quant. Grav. 21, 3803 (2004)

[8] S. Samuel, Phys. Rev. Lett. 90, id. 231101 (2003)

S. Samuel, Int. J. Mod. Phys. D 13, 1753 (2004)

[9] J.-F. Pascual-Sánchez, Int. J. Mod. Phys. D 13, 2345 (2004)

[10] S. Carlip, Phys. Lett. A 267, 81 (2000)

[11] J. D. Jackson, Classical Electrodynamics (John Wiley, New York, 1999)

[12] R. P. Feynman, et al., The Feynman Lectures on Physics (Addison-Wesley, Reading Massachusetts, 1970)

[13] T. Damour, In: Gravitational Radiation, eds. N. Deruelle and T. Piran, (North-Holland, Amsterdam, 1983) pp. 59-144

[14] S. M. Kopeikin and E. B. Fomalont, In: Proc. 6th European VLBI Network Symposium on New Developments in VLBI Science and Technology, eds. E. Ros, R. W. Porcas, A. P. Lobanov and J. A. Zensus (Max-Planck-Institut für Radioastronomie, Bonn, 2002) pp. 49-52, e-print: gr-qc/0206022

[15] L. Bel, N. Deruelle, T. Damour, J. Ibanez and J. Martin, Gen. Rel. Grav. 13, 963 (1981)

[16] S. M. Kopeikin and G. Schäfer, Phys. Rev. D 60, id. 124002 (1999)

[17] F. A. E. Pirani, Lectures on general relativity Vol. 1. (Prentice-Hall, New Jersey, 1964) pp. 249-373

[18] S. M. Kopeikin, G. Schäfer, C. R. Gwinn and T. M. Eubanks, Phys. Rev. D 59, id. 84023 (1999)

[19] J. M. Weisberg and J. H. Taylor, In: Radio Pulsars ASP Conf. Ser. Vol. 302, eds. M. Bailes, D. J. Nice and S. E. Thorsett (Sheridan Book, Ann Arbor, 2003) pp. 93-98

[20] C. M. Will, Theory and Experiment in Gravitational Physics (Cambridge University Press, Cambridge, 1993)

[21] S. M. Kopeikin, Class. Quantum Grav. 21, 3251 (2004)

[22] M. Á. G. Bonilla and J. M. M. Senovilla, Phys. Rev. Lett 78, 783 (1997)

[23] B. Mashhoon, In: Reference Frames and Gravitomagnetism, eds. J. F. Pascual-Sánchez, L. Floría, A. San Miguel and F. Vicente (World Scientific, Singapore, 2001) pp. 121-132

[24] S. M. Kopeikin, Phys. Lett. A 312, 147 (2003)

[25] L. D. Landau and E. M. Lifshitz, The Classical Theory of Fields (Pergamon, Oxford, 1971)

[26] V. P. Frolov, In: Problems in the General Theory of Relativity and Theory of Group Representations, ed. N. G. Basov (Proc. Lebedev Physics Institute, Moscow, 1986) Vol. 96, pp. 73-185

[27] E. M. Standish and J. G. Williams, "Orbital ephemerides of the Sun, Moon, and Planets", In: Explanatory Supplement to the Astronomical Almanac Chapter 5, ed. P. K. Seidelmann, (U.S. Naval Observatory, Washington D.C., 2006)

[28] S. M. Kopeikin, Int. J. Mod. Phys. D 15, (2006) (in press) e-print: gr-qc/0507001

[29] S. M. Kopeikin, Int. J. Mod. Phys. D 15, (2006) (in press) e-print: gr-qc/0501001

[30] S. M. Kopeikin and E. B. Fomalont, Phys. Lett. A (2006) (in press) e-print: gr-qc/0310065

[31] S. M. Kopeikin and B. Mashhoon, Phys. Rev. D 65, id. 064025 (2002)

[32] I. I. Shapiro, Phys. Rev. Lett. 13, 789 (1964)

[33] S. A. Klioner, Astron. Astrophys 404, 783 (2003)

[34] S. V Babak and L. P. Grishchuk, Int. J. Mod. Phys. D 12, 1905 (2003)

[35] S. M. Kopeikin, Sov. Astron. 29, 516 (1985)

[36] L. P. Grishchuk and S. M. Kopeikin, "Equations of motion for isolated bodies with relativistic corrections including the radiation reaction force", In: IAU Symp. 114: Relativity in Celestial Mechanics and Astrometry, eds. J. Kovalevsky and V. A. Brumberg (Reidel, Dordrecht, 1986) pp. 19-34

[37] T. Damour, "The problem of motion in Newtonian and Einsteinian gravity", In: Three Hundred Years of Gravitation, eds. S. W. Hawking and W. Israel (Cambridge University Press, Cambridge, 1987) pp. 128-198

[38] G. D. Moore and A. E. Nelson, J. High Energy Phys. 0109, 023 (2001)

[39] H. Asada, Int. J. Mod. Phys. D bf 15, (2006) (in press)

[40] see http://www.ligo.caltech.edu/

[41] see http://lisa.jpl.nasa.gov/

[42] V. B. Braginskii, Physics-Uspekhi 46, 81 (2003)

[43] C. M. Will, "Has the speed of gravity been measured ?" http://wugrav.wustl.edu/people/CMW/SpeedofGravity.html (cited on March 24, 2006)

[44] A. Z. Petrov, Einstein Spaces (Pergamon, Oxford, 1969)

[45] S. Kopeikin, P. Korobkov and A. Polnarev, Class. Quant. Grav. (2006) (submitted) e-print: gr-qc/0603064

[46] S. Kopeikin and P. Korobkov, Gen. Rel. Grav. (2006) submitted e-print: gr-qc/0510084

[47] D. J. Griffiths, Introduction to Electrodynamics (Prentice Hall, New Jersey, 1999)

[48] O. J. Sovers and C. S. Jacobs, JPL Publication 83-39, Rev. 5, p6 (1994)

[49] J. D. Barrow and J. Levin, Phys. Rev. A 63, id. 044104 (2001)

[50] C. M. Will, Phys. Rev. D 67, id. 062003 (2003) 
[51] J. L. Synge, Relativity: The General Theory (North-Holland, Amsterdam, 1960)

[52] T. Jacobson and D. Mattingly, Phys. Rev. D 70, id. 024003 (2004)

[53] Ya. B. Zeldovich and A. D. Myskis, Elements of Mathematical Physics (Nauka, Moscow, 1973) (in Russian)

[54] C. M. Will, "The Confrontation between general relativity and experiment" Liv. Rev. Rel. 4 (2001) http://www.livingreviews.org/lrr-2001-4 (cited on November 10, 2005) 


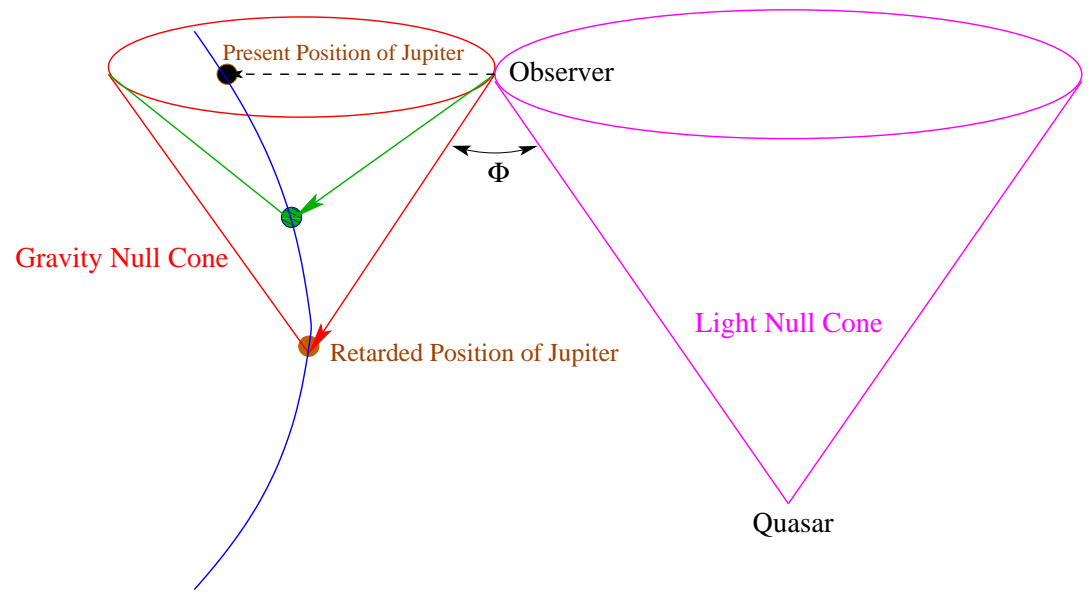

FIG. 1: The two null cones related to the experiment are shown. The gravity cone is a solution of the Einstein equations and describes null characteristics of gravity originating from moving Jupiter. The light cone is a solution of the Maxwell equations and describes the null characteristics of a radio wave from a quasar. The gravitational deflection of light is seen at the point where the gravity cone of Jupiter passes through the observer. VLBI measures the Minkowski dot product $\Phi=-k_{\alpha} r^{\alpha}$ between two null vectors $k^{\alpha}$ and $r^{\alpha}=x^{\alpha}-x_{J}^{\alpha}(s)$ at the point of observation directed to the observer from the quasar and from Jupiter, respectively. Had moving Jupiter not been detected at the retarded position on its world line, the speed of gravity would not be equal to the speed of light and the general theory of relativity would be violated. 


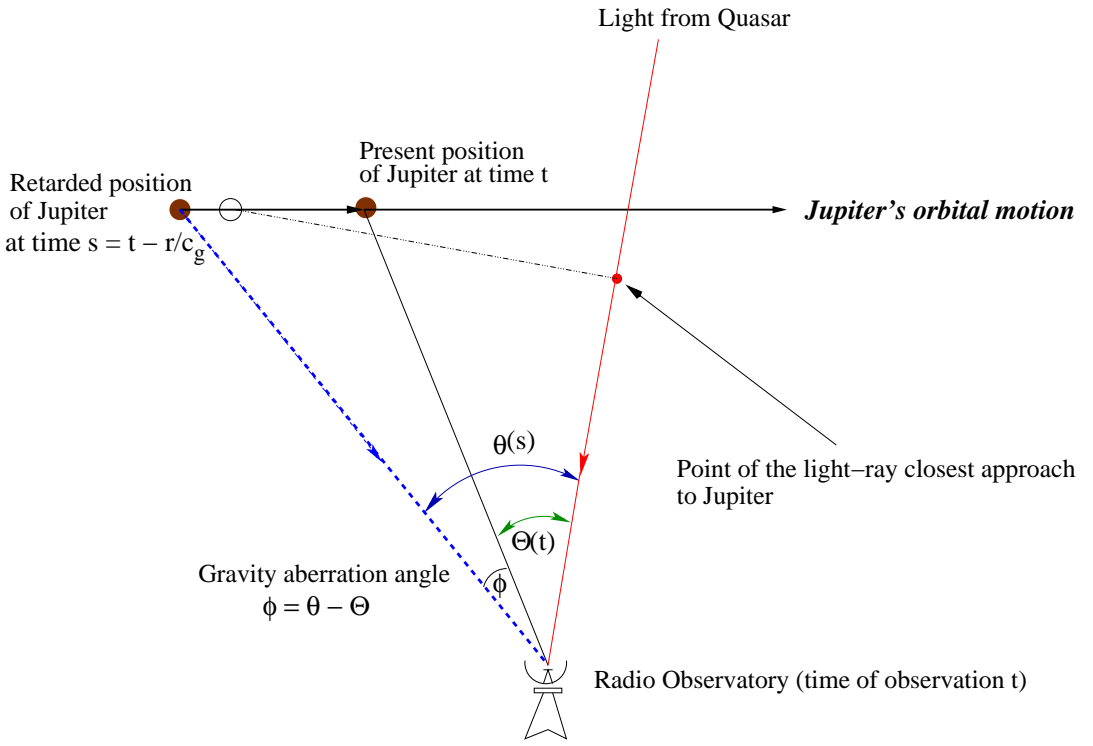

FIG. 2: Light propagates from the quasar toward a VLBI station (observer) on the Earth. While the light propagates, Jupiter is moving. General relativity predicts [1, 2] that the light observed at the time $t$ is deflected most strongly by Jupiter when it is located at the retarded position $\boldsymbol{x}_{J}\left(s_{g}\right)\left(s_{g}=t-r / c_{g}\right)$ for $c_{g}=c$, regardless of the direction of propagation of the light ray and the magnitude of the light-ray impact parameter with respect to Jupiter. This property of the gravitational deflection of light by a moving massive body can be used in order to measure the aberration of gravity force with respect to the aberration of light. The region of the sky which is imaged by the VLBI observations is smaller than the angle $\Theta$, so that radio emission of Jupiter is not detected when the quasar is observed. 


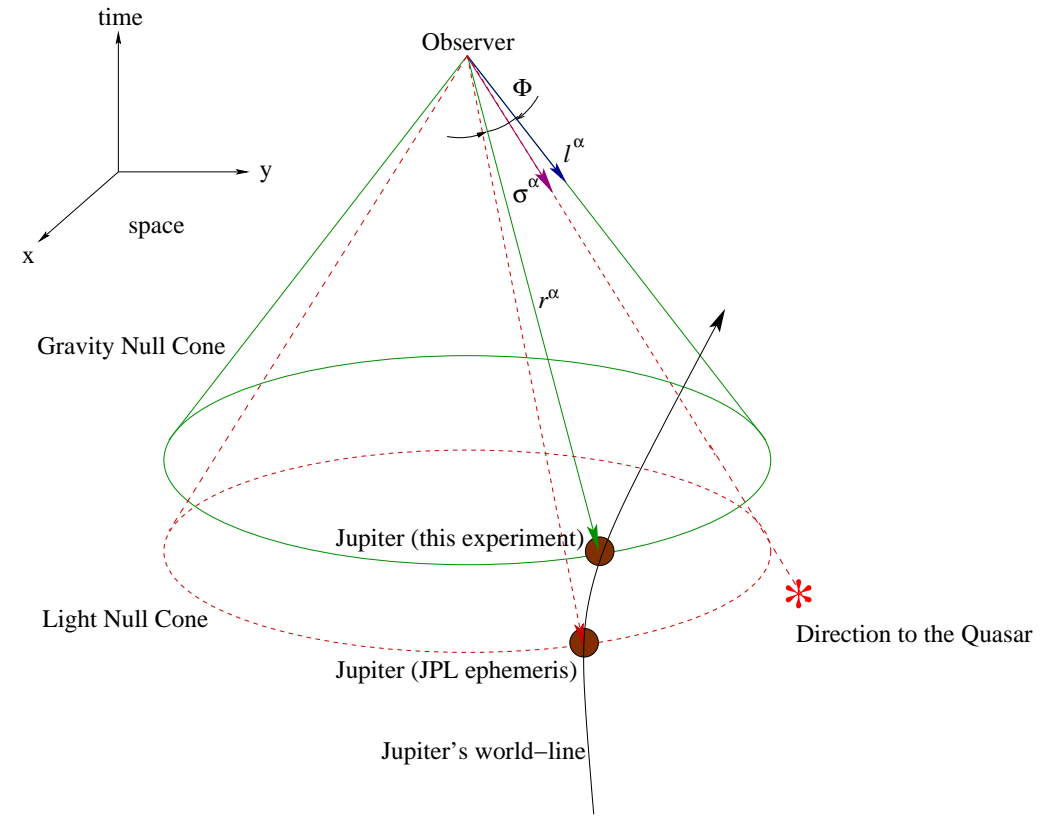

FIG. 3: The two null cones of the bi-metric theory are shown (see appendix). Retarded position of Jupiter due to the finite speed of gravity is on the gravity null-cone while Jupiter's radio position is on the light null-cone. The unperturbed direction to the quasar is defined by vector $\sigma^{\alpha}$ lying on the light null-cone of the optical metric $\bar{g}_{\alpha \beta}$. Gravity perturbs the light nullcone vector $\sigma^{\alpha}$ and changes its direction to the gravity null-cone vector $l^{\alpha}$. Gravitational field of moving Jupiter deflects the quasar's light from the retarded position defined with respect to observer by vector $r^{\alpha}=x^{\alpha}-x_{J}^{\alpha}(s)$ which lies on the gravity null-cone. Perturbed eikonal $\psi \simeq 2 M_{J} \ln \Phi$, where $\Phi=-l_{\alpha} r^{\alpha}$. Components of the vector $l^{\alpha}$ are calculated from vector $\sigma^{\alpha}$ and four-velocity of Jupiter $u^{\alpha}$ which are known. The angle $\Phi$ is calculated assuming that the retarded position of Jupiter is defined by the JPL ephemerides and lies on the light null-cone. The observed value of the angle $\Phi$ is fit to that defined by the JPL ephemerides by adjusting the parameter $\epsilon$ defining the difference between the speed of light and gravity. This procedure measures the speed of gravity with respect to light within the accuracy of $20 \%$ for $10 \mu$ as astrometric tolerance in observing small angles in the sky with the phase-referencing VLBI technique. 